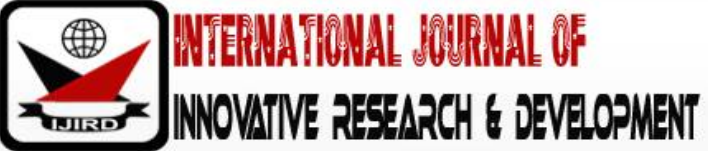

ISSN 2278 - 0211 (Online)

\section{Analysis of Physico-Chemical Properties of Soil in Relation to Arable Crop Production in Abia State, Nigeria}

Okorie Fidelis Chinazor
Lecturer, Department of Geography and Environmental Management
Institution name: Imo State University Owerri, Nigeria
Njoku, John Didacus
Associate Professor, Department of Environmental Technology
Federal University of Technology Owerri, Nigeria
Onweremadu Emmanuel Uzoma
Professor, Department of Soil Science and Technology
Federal University of Technology Owerri, Nigeria
Iwuji Martin Chidinma
Lecturer, Department of Environmental Technology
Federal University of Technology Owerri, Nigeria

\begin{abstract}
:
This study analyzed physicochemical properties of soil of Abia state Nigeria in relation to arable crop production. In the research, two (2) crops, Cassava and Maize were identified because of their positions among staple crops as well as relevance to the socio-economic well-being of the people of Abia sate and Nigeria in general. A free survey was employed for the study after the three zones in the state was purposely selected for representation of the soil sampling. Two types of sampling were conducted; Auger sampling was conducted at 0-20 and 20-40 cm depths for arable crop production purposes while soil profile sampling was done for in-depth characterization and scientific classification of soils. The zones include Ikwuano, Obuzo and Ovim. Soil samples were collected based on horizon differentiation. In each location, 10 auger samples of the same depth were collected and bulked to form a composite sample for laboratory analyses. Secondary data involved collection of annual crop yield data on maize and cassava for the sate over 30 years ranging from 1988 to 2017. The data were sourced from Agriculture Development Programme (ADP) headquarters in Umuahia. Soil data from auger samples were subjected to analysis of variance (ANOVA). Therefore, variation in properties among soil horizons were obtained using coefficient of variation. Also, the correlation coefficient ( $r$ ) and the coefficient of determination for simple linear regression $\left(\mathrm{r}^{2}\right)$ were calculated to determine the degree of association or relationship among some physico-chemical properties of the soils from selected profiles around the study area. Similarly, soil parameters were regressed against crop yield toestablish relationship existing among some soil properties and the crops. Results showed that at both $0-20 \mathrm{~cm}$ depth and $20-40 \mathrm{~cm}$ depth, the variations in the soil properties were from low to moderate and high. Therefore, comparing the two sampling depths, there was low variability in total sand, bulk density, total porosity, moisture content, field capacity, permanent wilting point and AWC. Sand and moisture content had medium variability while silt and clay had high variability. Also, there were different levels of variability among the soil chemical properties from $0-20 \mathrm{~cm}$ and $20-40 \mathrm{~cm}$ in the three zones. The results specifically showed that at both $0-20 \mathrm{~cm}$ and $20-40 \mathrm{~cm}$ depths, Ikwuano soils are more fertile than Obuzo and Ovim in terms of organic matter, organic carbon content, exchangeable bases and base saturation. However, the three locations need more external nutrient inputs for maximum crop production. The study showed that soils of Abia state have correlations with the arable crops, which means that soils properties affects crop production in the area at various levels. It was further observed that the soils contain more plant nutrient elements that will support crop production. However, the chemical properties of soils in the state are still low, there is therefore need to boost more the productivity of soils in the state for maximum crop production.
\end{abstract}

Keywords: Physico-chemical properties, soils, correlation, crop production, Abia state, Nigeria

\section{Introduction}

According to Gobin et al, (2004) Africa's soil resource has been deteriorating and will continue to deteriorate, probably due to changes in climate, land use and human activities in general Physical factors such as climate, topography, land cover and specific soil characteristics have important effects on the processes of soil erosion and soil formation which will in turn affect crop production. Edaphic factors presents major determinant in the growth and production of crops. Soil properties therefore, are among major physical environmental factors that can hinder or enhance crop production. 
Plants grown on land completely depend on soil on which they grow. More so, soil indicators which influence crop production are also affected by both natural and human induced factors. Soil factors affecting crop production include Soil moisture (water), Soil air, Soil temperature, Soil mineral matter, Soil Organic matter, Soil organisms and Soil reaction ( $\mathrm{pH})$, which is the $\mathrm{pH}$ (hydrogen ion concentration) of the soil.

The global food security crisis jeopardizes the lives of millions of people in vulnerable communities, particularly in Africa where poverty, malnutrition and death from hunger are rife (IPCC, 2007). There are indications and evidences that the agricultural and food system as well as the rural area across the world are experiencing major climatic changes (Apata et al., 2009). This change has drastically reduced soil fertility and led to poor agricultural outputs particularly in subSaharan Africa.

The recurrent food crisis in Nigeria is partly due to high rate of population growth over the food production level and erratic amounts of food crops produced from year to year. This however, can be attributed to high susceptibility of the country to serious environmental hazards extreme climate events, poor soil quality, deforestation, continuous cropping and unhindered desert encroachment (Ani, 2002).

Also Agboola and Ojeleye (2007) noted that the low-available water holding capacity of the soil result to poor crop growth in the tropics. In another study, Uchegbu (2006) believes that rising temperature affects soil moisture, which in turn could affect soil fertility. Supporting the above remark, Lia (1979) observed that the major agro-climatic constraints on agricultural production are related to insufficient, excessive or irregular moisture supply, which in turn will affect the length of growing period (LGP) of crops. From the foregoing, it is clear that there are many factors that affect the growing period of crops, which to a greater extent determine the crop growth cycle and its yield.

In this research, two (2) outstanding crops, Cassava and Maize have been identified because of their positions among staple crops as well as relevance to the socio-economic well-being of the people of Abia sate and Nigeria in general. This study is aimed to analyze physicochemical properties of soil of Abia state in relation to arable crop production with reference to maize and cassava.

\section{Area of Study}

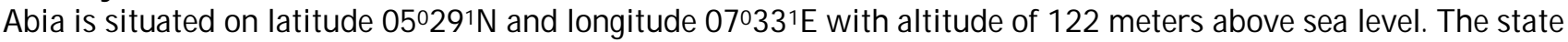
is located in the southeast geographical zone of Nigeria. It was carved out of the old Imo state on August 1991. The state is made of 17 local government areas (see figure 1), and the capital is at Umuahia while Aba is the commercial centre of the state. Rainfall is available in the state and evenly distributed all year round. The rainy season usually begins in March as moist Atlantic air mass known as the south-west monsoon invades the country and ends in October or early November. The dry season is from December to February. It witnesses two dry seasons, two wet seasons, if the little dry season "August break" is inclusive (Njoku, 2006). It has a tropical humid climate, with average temperature $32^{\circ} \mathrm{C}$ during dry season and $28^{\circ} \mathrm{C}$ in wet season.

The vegetation type is rainforest, (belonging rainforest vegetation zone of southeast Nigeria), being evergreen and luxuriant forest. Forest of the area is displayed in three layers - the ground layer, dominant middle layer and top layer. They are made up of three to six metre high herbs and shrubs, trees of 18 to 24 metres tall possessing robust branches and dark green foliage. Branches of one tree overlaps with those of others to create a continuous canopy of 30 to 60 metres high, some of the trees include iroko, obeche, palm trees, oil-bean trees and tropical cedar. Dependant species such as parasites, saprophytes, climbers and epiphytes are common in vegetation zone.

Since state is in tropical rainforest, the soils are largely a combination of hygromorphic soils, vertisols and ferralsols, and the vegetation plays the double role of supplying humus to the soil and protecting it from erosion. The area is well endowed with fossil fuel and other energy resources especially crude oil, natural gas, coal and biomass. The people of the state engage in subsistence, rural based, small scale farming and produce their own food crops. Therefore, rainfall availability and distribution is very essential in the area. Mineral resources found in the state include petroleum, natural gas, lead and zinc. The major tourist attractions are the National war Museum and Azumiri Blue River. According to 2006 Population and Housing Census, Abia state is made up of 1,430,298 males and 1,451,082 females, but currently, the state total population is estimated at 2,833,999 according to oasdom.com, (2019). 


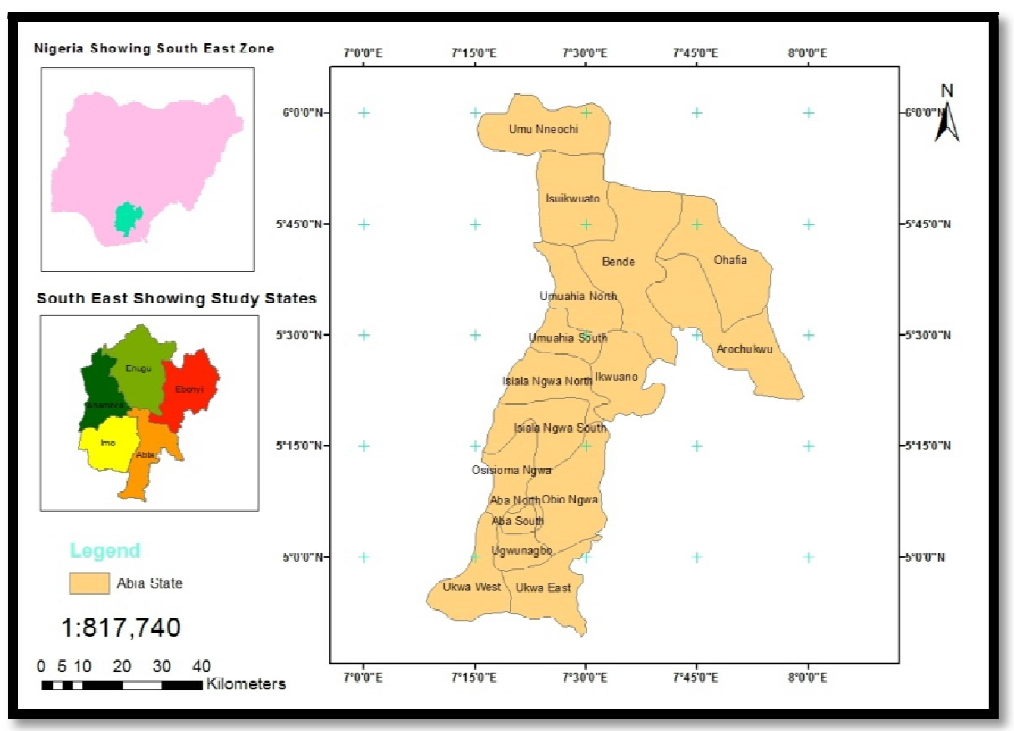

2.1. Materials and Methods

Figure 1: Abia State of Nigeria (Study Area) with Its Local Government Areas

The study involved both primary and secondary data sources and acquisitions.

\subsection{Primary Data Collection}

A free survey was employed for the study after the three zones in the state was purposely selected for representation of the soil sampling. The zones include Ikwuano, Obuzo and Ovim. The free survey was guided by size and extent of farming activities in each zone. Two types of sampling were conducted; Auger sampling was conducted at 0-20 and 20-40 cm depths for arable crop production purposes while soil profile sampling was done for in-depth characterization and scientific classification of soils.

Soil profile was dug, described and sampled using standard procedures as recommended by FAO (2006). Soil samples were collected based on horizon differentiation. Sampling started from the deepest horizon upwards in each soil profile. In each location, 10 auger samples of the same depth were collected and bulked to form a composite sample for laboratory analyses. Soil macro-morphological properties like horizon thickness and depth, soil colour, soil structure, soil consistence, boundary and others were performed in situ. Soil colour was determined under moist condition using Munsell Colour chart. Core soil samples were collected for bulk density determinations and the process is presented in plates 1 , while figure 3 shows checking and recording the soil colour.

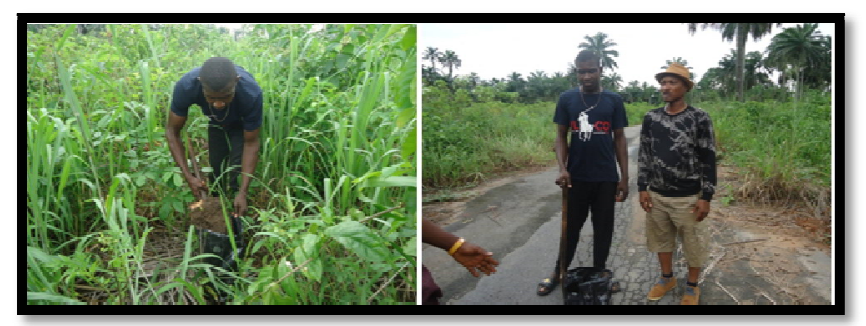

Figure 2: Soil Samples Collection during Field Studies Source: Author's Field Work 2019

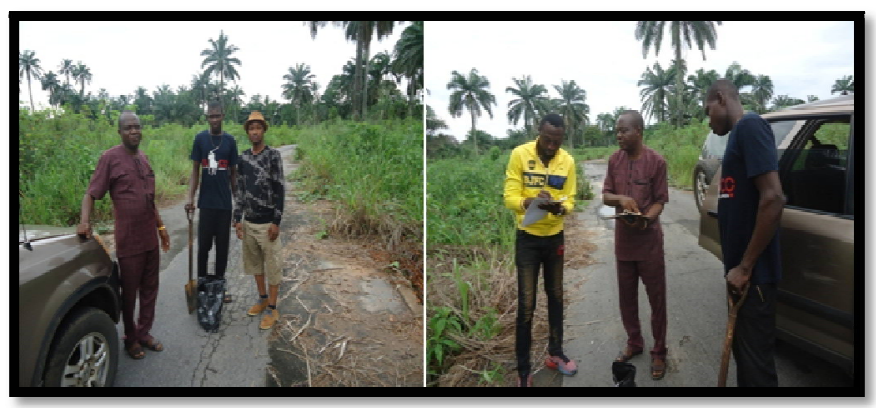

Figure 3: Checking Colour Chart and Recording Soil Colours in the Field

Source: Author's Field Work 2019 


\subsection{Secondary Data Collection}

The secondary data involved collection of annual crop yield data on maize and cassava (in kilograms/ hectare) for Abia sate over 30 years ranging from 1988 to 2017. The crop yield data were sourced from Agriculture Development Programme (ADP) headquarters in Umuahia. The crop yield data are presented in Tables 1. Also, both edaphic data and crop yield data were analyzed statistically to determine their relationship.

\begin{tabular}{|c|c|c|}
\hline Year & Maize & Cassava \\
\hline 1988 & 1.93 & 6.90 \\
\hline 1989 & 1.97 & 6.40 \\
\hline 1990 & 1.95 & 6.55 \\
\hline 1991 & 1.90 & 6.75 \\
\hline 1992 & 1.85 & 6.80 \\
\hline 1993 & 1.73 & 18.17 \\
\hline 1994 & 1.71 & 18.05 \\
\hline 1995 & 1.69 & 17.92 \\
\hline 1996 & 1.67 & 17.80 \\
\hline 1997 & 1.65 & 17.67 \\
\hline 1998 & 1.61 & 17.55 \\
\hline 1999 & 1.62 & 17.55 \\
\hline 2000 & 1.66 & 17.50 \\
\hline 2001 & 1.63 & 16.47 \\
\hline 2002 & 1.63 & 16.47 \\
\hline 2003 & 1.59 & 16.45 \\
\hline 2004 & 1.60 & 16.76 \\
\hline 2005 & 1.51 & 16.84 \\
\hline 2006 & 1.46 & 18.68 \\
\hline 2007 & 1.35 & 17.89 \\
\hline 2008 & 1.23 & 16.01 \\
\hline 2009 & 1.20 & 16.09 \\
\hline 2010 & 1.17 & 15.94 \\
\hline 2011 & 1.31 & 14.11 \\
\hline 2012 & 1.25 & 15.91 \\
\hline 2013 & 1.29 & 14.71 \\
\hline 2014 & 1.31 & 15.08 \\
\hline 2015 & 1.31 & 16.66 \\
\hline 2016 & 1.36 & 15.00 \\
\hline 2017 & 1.51 & 15.75 \\
\hline Total & 46.65 & 450.43 \\
\hline $1: C r o y$ & 5.5 & $1988-17)$ \\
\hline
\end{tabular}

Table 1: Crop Yield for Abia State in Metric Tons (1988-2017)

Source: ADP Umuahia, Abia State

\section{Laboratory Studies}

Soil samples were air-dried and sieved using 2-mm sieve. Particle size distribution was determined by hydrometer method (Gee and Or, 2002) while bulk density was measure by core procedure (Gross man and Reinsch, 2002). Results from particle size analysis (sand, silt and clay) values were used to obtained textural class using textural triangle. Bulk density values were used to calculate total porosity of soils given a relationship between bulk density and particle density (Foth, 1984).

Total Porosity (TP) $\stackrel{B D}{=\frac{B D}{P D}} \times \frac{100}{1}$

Where $\mathrm{BD}=$ determined bulk density

$\mathrm{PD}=$ particle density assured to be $2.65 \mathrm{mg} \mathrm{m}^{-3}\left(2.65 \mathrm{~g} / \mathrm{cm}^{-3}\right)$.

Gravitational moisture content ( $(\Theta \mathrm{m})$ was measured using the procedure as outlined in Obi (1990).

$\Theta \mathrm{m}=\frac{N S-D S}{D S} \times \frac{100 \%}{1}$

Where $\mathrm{m}=$ gravitmetric moisture content

Ws = weight of wet soil sample

Ds = weight of dry soil sample

$\mathrm{AWC}=\mathrm{FC}-\mathrm{PWP}$

Soil pH water and pH KCL were determined electronically in 1: 2.5 soil solutions! water- ratio (Hendershot et al., 1993).

Soil organic Carbon was measured by wet digestion using the procedure outlined in Soil Survey Staff (2010). Soil organic matter was calculated by multiplying organic carbon value by 1.724. Exchangeable basic cations were extracted using ammonium acetate at $\mathrm{pH}_{7}$. Therefore, exchangeable calcium and magnesium were determined using ethylene 
diamine-tetraascetic acid (EDTA) titration, and exchangeable potassium and sodium were determined by flame photometry (Soil Survey Staff, 2010). Exchangeable acidity (exchangeable hydrogen and aluminum) were measured by apparent titration (Soil Survey Staff, 2003). Cation exchange capacity (ACEC) was measured at pH of 7.0 (neutral) (Soil Survey Staff, 2010). Total nitrogen (TN) was determined by micro-kjedahl apparatus (Bremner, 1996). Available phosphorous was estimated by Bray 2 method according to procedure of Olson and Sommers (1982). Base saturation was computed as a sum of exchangeable basic cations ( $\mathrm{Ca}, \mathrm{Mg}, \mathrm{K}, \mathrm{Na}$ ) divided by Cation Exchange Capacity, multiplied by $100 \%$ (Soil Survey Staff, 2010).

\section{Date Analytical Techniques}

Soil data from auger samples were subjected to analysis of variance (ANOVA). Therefore, variation in properties among soil horizons were obtained using coefficient of variation. Also, the correlation coefficient ( $r$ ) and the coefficient of determination for simple linear regression $\left(\mathrm{r}^{2}\right)$ were calculated to determine the degree of association or relationship among some physico-chemical properties of the soils from selected profiles around the study area. Similarly, edaphic (soil) parameters (independent variables) were regressed against crop yield (dependent variables) to establish relationship existing among some soil properties and the crops.

\section{Results and Discussion}

\subsection{Physical Properties of Soils of Abia State}

Results of soil physical properties in Abia State are presented in Table 2. Results showed that at $0-20 \mathrm{~cm}$ depth, CS, FS, TS, Si and clay ranged from 350 - 500, 220 - 370, 700 - 720, 10 - 200 and 100 - $270 \mathrm{~g} / \mathrm{kg}$, respectively in the three studied location (Ikwuano, Obuzo and Ovim). Soil bulk density ranged from $1.22-1.25 \mathrm{~g} / \mathrm{cm}^{3}$ while total porosity ranged from 52.83 - $53.96 \%$. Moisture content ranged from 10.08 - 12.32\%. FC, PWP and AWC ranged from $0.198-0.217,0.082$ - 0.109, and $0.102-0.116 \mathrm{~g} / \mathrm{kg}$ respectively. There were low variations in fine sand, bulk density, total porosity, moisture content, field capacity, permanent wilting point and AWC while medium variations were observed on coarse sand and total sand.

At 20 - $40 \mathrm{~cm}$ depth, the values of CS, FS, TS, Si and clay ranged from $300-500,280-350,580-850,20-160$ and $90-200 \mathrm{~g} / \mathrm{kg}$ respectively. Bulk density and total porosity ranged from $1.31-1.36 \mathrm{~g} / \mathrm{cm}^{3}$ and $47.92-50.56 \%$. Filed capacity, PWP and AWC ranged from $0.209-0.218,0.079-0.101$ and $0.117-0.133 \mathrm{~g} / \mathrm{kg}$ respectively. There were low variations in bulk density, total porosity, moisture content, FC and PWP while there were moderated variations in FS, Cl, and AWC. High variations were observed in fine sand, bulk density, total porosity, field capacity, permanent wilting point and AWC. Medium variations were observed in coarse sand, total sand and moisture content while high variation were observed in clay fraction. Comparing the two sampling depths, there was low variability in total sand, bulk density, total porosity, moisture content, field capacity, permanent wilting point and AWC. Fine sand and moisture content had medium variability while silt and clay had high variability. These variations in the physical properties of Ikwuano, Obuzo and Ovim could be attributed to land use system.

\begin{tabular}{|c|c|c|c|c|c|c|c|c|c|c|c|c|}
\hline Location & CS & FS & TS & $\mathbf{S i}$ & $\mathrm{Cl}$ & TC & BD & TP & Ǿm & FC & PWP & AWC \\
\hline & $\mathrm{g} / \mathrm{kg}$ & $\mathbf{g} / \mathbf{k g}$ & $\mathrm{g} / \mathrm{kg}$ & $\mathrm{g} / \mathrm{kg}$ & $\mathrm{g} / \mathbf{k g}$ & & $\mathrm{g} / \mathrm{cm}^{3}$ & $\%$ & $\%$ & $\mathrm{~g} / \mathrm{kg}$ & $\mathbf{g} / \mathbf{k g}$ & $\mathrm{g} / \mathrm{kg}$ \\
\hline & & & & \multicolumn{3}{|c|}{$\frac{\text { Sampling depth }(0-}{20 \mathrm{~cm})}$} & & & & & & \\
\hline Ikwuano & 500 & 220 & 720 & 10 & 270 & SCL & 1.25 & 52.83 & 12.32 & 0.198 & 0.082 & 0.116 \\
\hline Obuzo & 350 & 370 & 720 & 180 & 100 & SL & 1.22 & 53.96 & 10.08 & 0.217 & 0.109 & 0.108 \\
\hline Ovim & 400 & 300 & 700 & 200 & 100 & SL & 1.24 & 53.2 & 10.46 & 0.206 & 0.104 & 0.102 \\
\hline Mean & 417 & 297 & 713 & 130 & 157 & & 1.24 & 53.33 & 10.95 & 0.207 & 0.098 & 0.109 \\
\hline $\mathrm{CV}(\%)$ & 18.3 & 1.6 & 25.3 & 80.3 & 62.6 & & 1.2 & 1.1 & 10.9 & 4.6 & 14.6 & 6.5 \\
\hline $\mathrm{SE}$ & 76.4 & 11.55 & 75.1 & 104.4 & 98.1 & & 0.02 & 0.58 & 1.19 & 0.009 & 0.01 & 0.01 \\
\hline & & & & \multicolumn{4}{|c|}{ Sampling depth $(20-40 \mathrm{~cm})$} & & & & & \\
\hline Ikwuano & 480 & 300 & 780 & 20 & 200 & SCL & 1.36 & 48.67 & 12.96 & 0.212 & 0.079 & 0.133 \\
\hline Obuzo & 500 & 350 & 850 & 160 & 90 & SL & 1.31 & 50.56 & 8.7 & 0.209 & 0.088 & 0.121 \\
\hline Ovim & 300 & 280 & 580 & 160 & 160 & SL & 1.38 & 47.92 & 10.2 & 0.218 & 0.101 & 0.117 \\
\hline Mean & 427 & 310 & 737 & 113 & 150 & & 1.35 & 49.05 & 10.62 & 0.213 & 0.089 & 0.124 \\
\hline $\mathrm{CV}(\%)$ & 25.8 & 11.6 & 19 & 71.3 & 37.1 & & 2.7 & 2.8 & 20.3 & 2.2 & 12.4 & 6.7 \\
\hline SE & 110.2 & 36.1 & 140.1 & 80.3 & 55.7 & & 0.04 & 1.36 & 2.16 & 0.004 & 0.11 & 0.01 \\
\hline Grand mean & 422 & 303 & 725 & 122 & 153 & & 1.29 & 51.19 & 10.79 & 0.210 & 0.094 & 0.116 \\
\hline CV(\%) & 22.5 & 19.4 & 13.7 & 76.7 & 52 & & 2.1 & 2 & 16.2 & 3.6 & 13.7 & 6.6 \\
\hline SE & 94.8 & 58.9 & 99.4 & 93.4 & 79.8 & & 0.03 & 1.05 & 1.75 & 0.01 & 0.01 & 0.01 \\
\hline
\end{tabular}

Table 2: Physical Properties of Soils of Abia State 


\subsection{Soil Chemical Properties of Soils of Abia State}

Soil chemical properties of Abia State are presented in Table 3. Results showed that within the $20 \mathrm{~cm}$ soil depth, soils of Ikwuano, Obuzo and Ovim are strongly acidic with pH in water ranging from 3.4 to 4.3 and $4.5-4.9$ in $\mathrm{KCl}$. Organic carbon and organic matter contents were low with the mean value 16.53 and $28.47 \mathrm{~g} / \mathrm{kg}$ respectively. Total nitrogen according to FAO (2006) rating was also low and ranged from $1.3-1.5 \mathrm{~g} / \mathrm{kg}$. Exchangeable bases were low with total using Esu (1991) rating. Total exchangeable bases ranged from 0.99 - 1.78 cmol.kg. Ikwuano location had higher total exchangeable bases $(3.70 \mathrm{cmol} / \mathrm{kg})$ while effective cation exchange capacity and base saturation had the values at Obuzo location. ACEC ranged from $5.38-7.82 \mathrm{cmol} / \mathrm{kg}$. Available phosphorus was low and ranged from $8.80-25.20 \mathrm{mg} / \mathrm{kg}$.

At $20-40 \mathrm{~cm}$ auger depth, soil pH range in water and $\mathrm{KCl}$ was $3.2-4.1$ and $4.1-4$. 6. Organic carbon and organic matter ranged from $8.6-10.0$ and $14.8-17.2 \mathrm{~g} / \mathrm{kg}$. The nitrogen content was low with the range of $0.9-1.1 \mathrm{~g} / \mathrm{kg}$. Exchangeable cations ( $\mathrm{Ca}, \mathrm{Mg}$, $\mathrm{K}$ and $\mathrm{Na}$ ) were low in the three locations according to FAO (2006) though with the highest values found at Obuzo village. Total exchangeable acidity ranged from $3.2-4.0 \mathrm{cmol} / \mathrm{kg}$ while effective cation exchange capacity was also low with the highest values found at Ikwuano. Base saturation was low and available phosphorus ranged from 4.8 - 13.6 $\mathrm{mg} / \mathrm{kg}$. There were also different levels of variability among the soil chemical properties from $0-20 \mathrm{~cm}$ and $20-40 \mathrm{~cm}$. These results showed that at both $0-20 \mathrm{~cm}$ and $20-40 \mathrm{~cm}$ depths, Ikwuano soils are more fertile than Obuzo and Ovim in terms of organic matter, organic carbon content, exchangeable bases and base saturation. However, the three locations need more external nutrient inputs for maximum crop production.

\begin{tabular}{|c|c|c|c|c|c|c|c|c|c|c|c|c|c|c|c|c|c|}
\hline 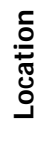 & $\begin{array}{l}\hat{g} \\
\text { 噌 }\end{array}$ & $\begin{array}{l}\widehat{\partial} \\
\hat{d}\end{array}$ & 8 & $\sum$ & Z & 8 & $\sum$ & $\forall$ & Z & 圆 & $\Xi$ & ₹ & 国 & 兽 & 8 & $\overrightarrow{8}$ & $\frac{4}{4}$ \\
\hline & & & $\mathrm{g} / \mathrm{kg}$ & $\mathrm{g} / \mathrm{kg}$ & $\mathrm{g} / \mathrm{kg}$ & 4 & & - & & $\mathrm{Cmol} / \mathrm{kg}$ & & & & 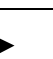 & & $\%$ & $\mathrm{Mg} / \mathrm{kg}$ \\
\hline & & & & & & \multicolumn{4}{|c|}{$\begin{array}{l}\text { Sampling depth }(0- \\
20 \mathrm{~cm})\end{array}$} & & & & & & & & \\
\hline 栗 & $\stackrel{m}{+}$ & $\stackrel{9}{+}$ & $\stackrel{H}{-}$ & $\stackrel{\overrightarrow{+}}{\stackrel{+}{+}}$ & $\stackrel{+}{-1}$ & - & ก๊ & $\stackrel{8}{0}$ & $\stackrel{1}{\circ}$ & 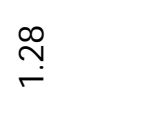 & $\stackrel{n}{-}$ & $\stackrel{+}{i}$ & $\hat{m}$ & $\begin{array}{l}\stackrel{\infty}{\sigma} \\
+\end{array}$ & ़ֻ & $\stackrel{\stackrel{2}{N}}{ }$ & $\begin{array}{l}\infty \\
\text { ì }\end{array}$ \\
\hline 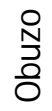 & $\stackrel{H}{m}$ & $\stackrel{L}{\leftrightarrow}$ & กิ & $\stackrel{?}{\stackrel{m}{m}}$ & $\stackrel{\text { ?ִ }}{\sim}$ & $\stackrel{m}{-}$ & $\stackrel{n}{0}$ & $\ddot{0}$ & $\overbrace{0}^{+}$ & $\stackrel{+H}{\stackrel{H}{-1}}$ & $\stackrel{N}{-}$ & $\stackrel{\sim}{N}$ & $\stackrel{\oplus}{m}$ & $\underset{⿱ ㇒}{\stackrel{H}{H}}$ & 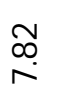 & $m$ & ヘึ \\
\hline व्द & + & $\stackrel{\infty}{+}$ & $\stackrel{\varphi}{\stackrel{\leftrightarrow}{\rightarrow}}$ & $\stackrel{+}{\stackrel{n}{n}}$ & $\stackrel{m}{-}$ & $\stackrel{0}{0}$ & ก̋ & $\stackrel{\overrightarrow{1}}{\overrightarrow{0}}$ & 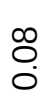 & @̊ & $\stackrel{m}{\rightarrow}$ & $\stackrel{\circ}{-}$ & is & $\begin{array}{l}\infty \\
\text { ஸे }\end{array}$ & $\begin{array}{l}\text { लొ } \\
\text { மึ }\end{array}$ & $\stackrel{\llcorner}{N}$ & $\begin{array}{l}\infty \\
\infty\end{array}$ \\
\hline 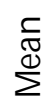 & ஓ, & $\stackrel{M}{\stackrel{M}{\not}}$ & 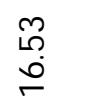 & $\begin{array}{l}\text { f! } \\
\stackrel{\infty}{N}\end{array}$ & $\stackrel{\circ}{\stackrel{0}{+}}$ & م̊ & ஸ̂. & : & $\stackrel{L}{\circ}$ & $\stackrel{m}{m}$ & กิ & $\hat{\circ}$ & $\begin{array}{c}m \\
m\end{array}$ & $\hat{\hat{\sigma}}$ & $\begin{array}{l}\stackrel{L}{\infty} \\
0 \\
0\end{array}$ & $\begin{array}{l}\hat{0} \\
\stackrel{\mathrm{N}}{0}\end{array}$ & $\begin{array}{l}\text { O̦ } \\
\infty \\
\infty \\
-1\end{array}$ \\
\hline$\frac{\delta}{\grave{b}}$ & $\stackrel{\infty}{\stackrel{\infty}{-}}$ & $\stackrel{\leftrightarrow}{+}$ & $\widehat{\stackrel{N}{N}}$ & $\hat{o}$ & $\stackrel{-}{\pi}$ & $\begin{array}{l}m \\
\text { فे }\end{array}$ & $\stackrel{\text { ஸे }}{N}$ & 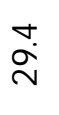 & 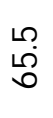 & $\begin{array}{l}m \\
\stackrel{\infty}{N}\end{array}$ & $\stackrel{\varphi}{+}$ & ¿্ঠ & $\underset{\sim}{\stackrel{-}{\sim}}$ & $\stackrel{\varphi}{\underset{H}{+}}$ & $\begin{array}{c}m \\
\infty \\
\rightarrow\end{array}$ & $\hat{\mathscr{\theta}}$ & $\begin{array}{l}\infty \\
\dot{\varphi} \\
\dot{\gamma}\end{array}$ \\
\hline 댄 & 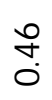 & సָ & $\underset{+}{\stackrel{H}{+}}$ & $\stackrel{\infty}{\stackrel{\infty}{\infty}}$ & $\overrightarrow{0}$ & $\stackrel{\stackrel{m}{0}}{0}$ & 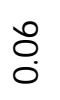 & $\stackrel{\text { Oे. }}{0}$ & $\stackrel{0}{0}$ & $\stackrel{\substack{m \\
m}}{0}$ & $\stackrel{\varphi}{0}$ & $\stackrel{\text { I }}{\circ}$ & $\stackrel{+}{0}$ & 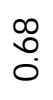 & $\stackrel{\text { N̦}}{\rightarrow}$ & $\underset{+}{\tilde{H}}$ & $\begin{array}{l}0 \\
\infty \\
\infty \\
\infty\end{array}$ \\
\hline & & & & & & \multicolumn{6}{|c|}{ 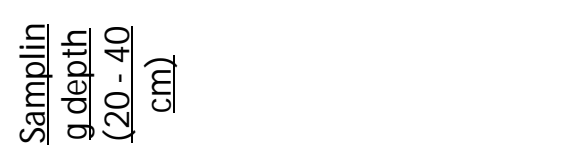 } & & & & & & \\
\hline 总 & $\stackrel{+}{+}$ & $\stackrel{\varphi}{\leftrightarrow}$ & $a$ & $\stackrel{\stackrel{L}{\circ}}{\stackrel{L}{\sim}}$ & 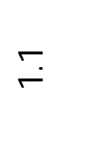 & $\stackrel{0}{0}$ & $\stackrel{-}{0}$ & $\stackrel{m}{0}$ & $\stackrel{\sigma}{0}$ & $\begin{array}{l}\text { + } \\
0 \\
0\end{array}$ & $\stackrel{\text { L }}{\rightarrow}$ & $\stackrel{\llcorner}{\sim}$ & 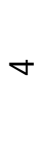 & $\underset{+}{\stackrel{+}{+}}$ & $\underset{\sim}{\stackrel{\infty}{\sim}}$ & $\stackrel{\llcorner}{\sim}$ & $\stackrel{\varphi}{\stackrel{\oplus}{\rightarrow}}$ \\
\hline
\end{tabular}




\begin{tabular}{|c|c|c|c|c|c|c|c|c|c|c|c|c|c|c|c|c|c|}
\hline 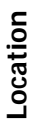 & 番 & 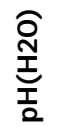 & 8 & $\sum$ & $Z$ & है & $\sum$ & $\underline{z}$ & 2 & 周 & \pm & ¿ & $\$$ & 曽 & $\overbrace{4}$ & $\vec{~}$ & $\frac{4}{8}$ \\
\hline 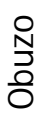 & ஸे & $\stackrel{\vec{F}}{+}$ & $\stackrel{\circ}{\rightarrow}$ & $\underset{\sim}{\stackrel{N}{\sim}}$ & ?ִ & $\stackrel{\infty}{0}$ & "̈) & $\stackrel{0}{0}$ & $\stackrel{\sigma}{0}$ & مิ & $\stackrel{m}{\rightarrow}$ & $\stackrel{\leftrightarrow}{i}$ & $\hat{m}$ & $\begin{array}{l}\hat{\theta} \\
+\end{array}$ & $\stackrel{m}{\circ}$ & 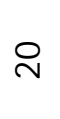 & $\stackrel{\llcorner 0}{\rightarrow}$ \\
\hline$\frac{q}{\delta}$ & $\stackrel{\infty}{m}$ & $\stackrel{+H}{+}$ & $\stackrel{\infty}{\infty}_{\infty}^{0}$ & 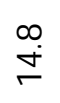 & ت] & $\stackrel{+}{0}$ & -1. & $\stackrel{1}{0}$ & $\stackrel{\text { Oִ }}{0}$ & $\stackrel{\varphi}{\circ}$ & $\stackrel{+}{-}$ & $\stackrel{\infty}{-}$ & ஸे & $\stackrel{\infty}{\dot{m}}$ & $\underset{\sim}{\stackrel{7}{\circ}}$ & $\stackrel{\infty}{\sim}$ & $\stackrel{\infty}{+\infty}$ \\
\hline $\begin{array}{l}\text { : } \\
\sum^{\infty}\end{array}$ & $\begin{array}{l}\stackrel{R}{2} \\
\text { m }\end{array}$ & $\hat{m}$ & $\begin{array}{l}\text { तิ } \\
\text { ஸे }\end{array}$ & $\begin{array}{l}\infty \\
\infty \\
\stackrel{\omega}{\sim}\end{array}$ & $\underset{\text { Oִ }}{\infty}$ & $\stackrel{8}{\bullet}$ & $\begin{array}{l}\stackrel{0}{-1} \\
\stackrel{0}{0}\end{array}$ & $\stackrel{L}{\circ}$ & 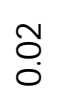 & 今. & $\underset{\rightarrow}{q}$ & 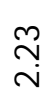 & $\begin{array}{l}\tilde{6} \\
\dot{m}\end{array}$ & $\begin{array}{l}\text { 움 } \\
\text { in }\end{array}$ & $\underset{6}{\mathscr{H}}$ & $\begin{array}{l}\hat{0} \\
\stackrel{-}{\sim}\end{array}$ & 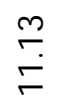 \\
\hline$\frac{\partial}{\frac{\partial}{b}}$ & $\underset{\sim}{\stackrel{H}{*}}$ & $\begin{array}{l}\infty \\
\stackrel{\infty}{0}\end{array}$ & $\stackrel{\infty}{\sim}$ & $\stackrel{\infty}{\wedge}$ & 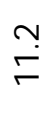 & $\ddot{m}$ & 0 & ஜి & $\begin{array}{l}\text { \%ి } \\
\text { هి }\end{array}$ & 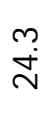 & $\vec{r}$ & $\stackrel{ન}{\neg}$ & $\underset{ت}{ت}$ & 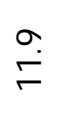 & $\underset{\sim}{\stackrel{\infty}{\sim}}$ & $\underset{\underset{H}{*}}{\stackrel{N}{*}}$ & $\hat{\sigma}$ \\
\hline 뎋 & ?̊ & $\stackrel{\stackrel{\llcorner}{N}}{0}$ & $\stackrel{\frac{1}{2}}{\stackrel{0}{0}}$ & 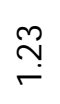 & $\stackrel{0}{-1}$ & ִָ & 0 & 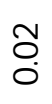 & $\tilde{o}_{0}^{\circ}$ & $\stackrel{\circ}{\circ}$ & $\ddot{0}$ & ֻొ & $\stackrel{+!}{0}$ & గి & $\stackrel{\text { L }}{\rightarrow}$ & ஸ̂ & 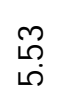 \\
\hline 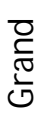 & $\begin{array}{l}\infty \\
\infty \\
\text { in }\end{array}$ & $\begin{array}{l}\stackrel{L}{\llcorner} \\
+ \\
+\end{array}$ & $\begin{array}{l}\hat{\infty} \\
\stackrel{\leftrightarrow}{\sim} \\
\stackrel{-}{1}\end{array}$ & $\begin{array}{l}\stackrel{L}{\mathrm{~N}} \\
\stackrel{\mathrm{N}}{\mathrm{N}}\end{array}$ & $\underset{\text { N̦ }}{-}$ & 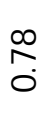 & $\stackrel{-}{\circ}$ & $\stackrel{\hat{O}}{0}$ & Oి & نْ & $\stackrel{M}{M}$ & $\stackrel{\sim}{\stackrel{\sim}{i}}$ & $\begin{array}{l}\stackrel{\infty}{+} \\
\text { ले }\end{array}$ & $\begin{array}{l}\text { ヴ } \\
+\forall\end{array}$ & $\begin{array}{l}0 \\
0 \\
0\end{array}$ & $\begin{array}{l}\hat{\theta} \\
\hat{i} \\
\hat{N}\end{array}$ & $\begin{array}{l}\text { Oे } \\
\stackrel{\rho}{\circ}\end{array}$ \\
\hline 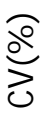 & $\underset{\sim}{\stackrel{\sim}{\sim}}$ & $\overrightarrow{\dot{\omega}}$ & $\begin{array}{l}\text { ֻें } \\
\text { ते }\end{array}$ & $\begin{array}{l}\stackrel{H}{~} \\
\stackrel{\leftrightarrow}{0}\end{array}$ & @) & 吕 & $\stackrel{\stackrel{\Omega}{+}}{\stackrel{\text { N }}{N}}$ & ஸे & 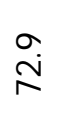 & $\begin{array}{l}\stackrel{m}{\infty} \\
\stackrel{\infty}{N}\end{array}$ & $\overrightarrow{6}$ & 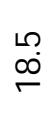 & $\begin{array}{l}\stackrel{0}{-} \\
\stackrel{-}{-}\end{array}$ & $\stackrel{\stackrel{H}{M}}{\stackrel{m}{\rightarrow}}$ & 敬 & $\stackrel{\leftrightarrow}{\bullet}$ & హ) \\
\hline 步 & $\stackrel{0}{\stackrel{0}{0}}$ & 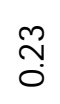 & ले & 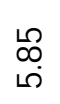 & $\stackrel{\overrightarrow{1}}{\overrightarrow{0}}$ & $\stackrel{+}{\oplus}$ & ర্. & $\stackrel{\text { Oִ }}{0}$ & $\stackrel{1}{0}$ & $\stackrel{m}{0}$ & $\stackrel{\infty}{\infty}$ & $\stackrel{+!}{0}$ & $\overbrace{0}^{+}$ & $\stackrel{\overrightarrow{0}}{0}$ & $\stackrel{\text { }}{\stackrel{\leftrightarrow}{\sim}}$ & $\underset{\dot{m}}{\stackrel{N}{N}}$ & 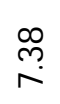 \\
\hline
\end{tabular}

Table 3: Soil Chemical Properties of Soils of Abia State

\subsection{Soil Physical Properties of Ikwuano, Umuahia Abia State Profile}

The physical properties of Ikwuano in Abia State are presented in Table 4. The textural class of the soils was silt loam and silt clay loam. The mean value of CS, FS, TS, Si and Cl were 680, 104, 764, 20 and 196g/ $\mathrm{kg}$ respectively. Average value of bulk density and total porosity was $1.38 \mathrm{~g} / \mathrm{cm}^{3}$ and $47.99 \%$. Moisture content increased down the depth with mean value of 13.18. Field capacity and permanent wilting pointing had the mean values of 0.226 and $0.112 \mathrm{~g} / \mathrm{kg}$ respectively while AWC had mean value of $0.114 \mathrm{~g} / \mathrm{kg}$.

Variations occurred among soil physical properties from A to BC horizon. Coarse sand, total sand, bulk density, total porosity, field capacity, PWP and AWC exhibited low variability while clay had low variation and fine sand and silt exhibited high variation using Wilding (1996) rating. 


\begin{tabular}{|c|c|c|c|c|c|c|c|c|c|c|c|c|c|}
\hline Horizon & $\begin{array}{c}\text { depth } \\
\text { (cm) }\end{array}$ & CS & FS & TS & $\overline{\mathrm{Si}}$ & $\mathrm{Cl}$ & TC & BD & TP & Ǿm & FC & PWP & $\overline{\text { AWC }}$ \\
\hline & & $\mathrm{g} / \mathrm{kg}$ & $\mathrm{g} / \mathrm{kg}$ & $\mathrm{g} / \mathrm{kg}$ & $\mathrm{g} / \mathrm{kg}$ & $\mathrm{g} / \mathrm{kg}$ & & $\underset{3}{\mathrm{~g} / \mathrm{cm}}$ & $\%$ & $\%$ & $\mathrm{~g} / \mathrm{kg}$ & $\mathrm{g} / \mathrm{kg}$ & $\mathrm{g} / \mathrm{kg}$ \\
\hline $\mathrm{A}$ & $0-11$ & 600 & 210 & 710 & 20 & 170 & SL & 1.21 & 54.33 & 12.9 & $\begin{array}{c}0.22 \\
3\end{array}$ & $\begin{array}{c}0.10 \\
4\end{array}$ & $\begin{array}{c}0.11 \\
9\end{array}$ \\
\hline$\overline{\mathrm{AB}}$ & $11-39$ & 700 & 100 & 800 & 10 & 190 & SL & 1.33 & 49.81 & 13 & $\begin{array}{c}0.22 \\
5\end{array}$ & $\begin{array}{c}0.11 \\
9\end{array}$ & $\begin{array}{c}0.10 \\
6\end{array}$ \\
\hline Bt1 & $\begin{array}{l}39- \\
105\end{array}$ & 600 & 100 & 700 & 10 & 290 & SCL & 1.38 & 47.92 & $\begin{array}{c}14.6 \\
8\end{array}$ & $\begin{array}{c}0.23 \\
1\end{array}$ & $\begin{array}{c}0.10 \\
7\end{array}$ & $\begin{array}{c}0.12 \\
4\end{array}$ \\
\hline Bt2 & $\begin{array}{c}105- \\
170\end{array}$ & 700 & 80 & 780 & 30 & 190 & $\mathrm{SL}$ & 1.46 & 44.9 & $\begin{array}{c}13.1 \\
2\end{array}$ & $\begin{array}{c}0.22 \\
7\end{array}$ & 0.11 & $\begin{array}{c}0.11 \\
7\end{array}$ \\
\hline $\mathrm{BC}$ & $\begin{array}{c}170- \\
265\end{array}$ & 800 & 30 & 830 & 30 & 140 & $\mathrm{SL}$ & 1.51 & 43.01 & 12.2 & $\begin{array}{c}0.22 \\
4\end{array}$ & $\begin{array}{c}0.12 \\
1\end{array}$ & $\begin{array}{c}0.10 \\
3\end{array}$ \\
\hline Mean & & 680 & 104 & 764 & 20 & 196 & & 1.378 & $\begin{array}{c}47.99 \\
4\end{array}$ & $\begin{array}{c}13.1 \\
8\end{array}$ & $\begin{array}{c}0.22 \\
6\end{array}$ & $\begin{array}{c}0.11 \\
2\end{array}$ & $\begin{array}{c}0.11 \\
4\end{array}$ \\
\hline $\mathrm{CV}(\%)$ & & 12.3 & 63.3 & 7.4 & 50 & 28.8 & & 8.5 & 9.2 & 6.9 & 1.4 & 6.7 & 7.8 \\
\hline $\mathrm{SE}$ & & 83.7 & 65.8 & 56.8 & 10 & 56.4 & & 0.12 & 4.41 & 0.91 & $\begin{array}{c}0.00 \\
3\end{array}$ & 0.01 & 0.01 \\
\hline
\end{tabular}

Table 4: Soil Physical Properties of Ikwuano, Umuahia Abia State Profile

\subsection{Soil Physical Properties of Obuzo, Abia State Profile}

Table 5 showed the physical properties of Obuzo soil, Abia State. Results showed that the mean values of coarse sand, fine sand, total sand, silt and clay were $397,215,645,167$ and $222 \mathrm{~g} / \mathrm{kg}$ respectively. The textural class of the soil from A to AB horizon was silt loam; from Bt1 to Bt3 was silt clay loam while at Bt4 the texture was silt loam. Bulk density increased with increase in depth while total porosity decreased with depth. The average bulk density and total porosity were $1.39 \mathrm{~g} / \mathrm{cm}^{3}$ and $47.61 \%$. Moisture content increased with depth with an average value of $13.42 \%$. Filed capacity and permanent wilting point had the mean value of 0.212 and $0.103 \mathrm{~g} / \mathrm{kg}$ respectively. There was downward increase in the AWC down the pit. In all the properties, there are various degrees of variability. Apart from bulk density, total porosity, field capacity, PWP and AWC, other physical properties showed medium to high variation.

\begin{tabular}{|c|c|c|c|c|c|c|c|c|c|c|c|c|c|}
\hline Horizon & $\begin{array}{c}\text { depth } \\
\text { (cm) }\end{array}$ & CS & FS & TS & $\mathbf{S i}$ & $\mathrm{Cl}$ & TC & BD & TP & Ǿm & FC & PWP & AWC \\
\hline & & $\mathrm{g} / \mathrm{kg}$ & $\mathrm{g} / \mathrm{kg}$ & $\mathrm{g} / \mathrm{kg}$ & g/ kg & g/ kg & & $\mathrm{g} / \mathrm{cm}^{3}$ & $\%$ & $\%$ & $\mathrm{~g} / \mathrm{kg}$ & $\mathrm{g} / \mathrm{kg}$ & $\mathrm{g} / \mathrm{kg}$ \\
\hline A & $0-12$ & 400 & 320 & 720 & 150 & 130 & SL & 1.18 & 55.47 & 11.44 & 0.219 & 0.109 & 0.11 \\
\hline$\overline{\mathrm{AB}}$ & $12-28$ & 380 & 320 & 800 & 150 & 150 & SL & 1.27 & 52.07 & 12.6 & 0.211 & 0.103 & 0.108 \\
\hline Bt1 & $28-54$ & 400 & 200 & 700 & 100 & 300 & SCL & 1.38 & 47.92 & 14.7 & 0.213 & 0.099 & 0.114 \\
\hline Bt2 & $54-88$ & 300 & 200 & 500 & 100 & 400 & SCL & 1.44 & 45.66 & 14.8 & 0.216 & 0.098 & 0.118 \\
\hline Bt3 & $88-150$ & 400 & 150 & 550 & 250 & 200 & $\overline{\mathrm{SCL}}$ & 1.5 & 43.39 & 13.76 & 0.212 & 0.11 & 0.102 \\
\hline Bt4 & $\begin{array}{c}150- \\
190\end{array}$ & 500 & 100 & 600 & 250 & 150 & $\mathrm{SL}$ & 1.56 & 41.13 & 13.2 & 0.201 & 0.1 & 0.101 \\
\hline Mean & & 397 & 215 & 645 & 167 & 222 & & 1.39 & 47.61 & 13.42 & 0.212 & 0.103 & 0.109 \\
\hline $\mathrm{CV}(\%)$ & & 16.1 & 41.6 & 17.6 & 41 & 16.1 & & 10.3 & 11.3 & 9.6 & 2.9 & 5 & 6.1 \\
\hline $\mathrm{SE}$ & & 63.8 & 89.4 & 113.8 & 68.3 & 63.8 & & 0.14 & 5.39 & 1.29 & 0.01 & 0.005 & 0.01 \\
\hline
\end{tabular}

Table 5: Soil Physical Properties of Obuzo, Abia State Profile

5.5. Soil Physical Properties of Ovim, Abia State Profile

The physical properties of soils in Ovim Abia State are shown in Table 6. The epidepon is dominated by sand fraction. The mean values of CS, FS, TS, Si and Cl are 440, 98, 538, 128 and $326 \mathrm{~g} / \mathrm{kg}$ respectively. These properties showed medium variation expect clay fraction that showed high variability down the horizons. These variations gave rise to three textural classes. The mean values of bulk density, total porosity, and moisture contents were $1.40 \mathrm{~g} / \mathrm{cm}^{3}, 47.01 \%$ and $13.95 \mathrm{~g} / \mathrm{kg}$. The moisture content was lowest at the epipedon and this could be due to high sand fraction recorded on the top soil. Mean values of field capacity, PWP and AWC are 0.229, 0.11 and $0.117 \mathrm{~g} / \mathrm{kg}$ respectively. Bulk density, total porosity, moisture content, field capacity, PWP and AWC showed low variations. 


\begin{tabular}{|c|c|c|c|c|c|c|c|c|c|c|c|c|c|}
\hline Horizon & $\begin{array}{c}\text { depth } \\
\text { (cm) }\end{array}$ & CS & FS & TS & $\overline{\mathrm{Si}}$ & $\mathrm{Cl}$ & TC & BD & TP & Ǿm & FC & PWP & AWC \\
\hline & & $\mathrm{g} / \mathrm{kg}$ & $\mathbf{g} / \mathbf{k g}$ & $\mathbf{g} / \mathbf{k g}$ & g/ kg & $\mathrm{g} / \mathrm{kg}$ & & $\mathbf{g} / \mathbf{c m}^{3}$ & $\%$ & $\%$ & $\mathrm{~g} / \mathrm{kg}$ & $\mathbf{g} / \mathbf{k g}$ & $\mathrm{g} / \mathrm{kg}$ \\
\hline $\mathrm{A}$ & $0-17$ & 600 & 120 & 720 & 160 & 130 & SL & 1.2 & 54.72 & 10.6 & 0.208 & 0.102 & 0.106 \\
\hline $\mathrm{AB}$ & $17-42$ & 500 & 70 & 570 & 130 & 300 & SCL & 1.36 & 48.67 & 14.69 & 0.224 & 0.112 & 0.112 \\
\hline Bt1 & $42-81$ & 400 & 100 & 500 & 100 & 400 & SCL & 1.43 & 46.03 & 14.82 & 0.236 & 0.113 & 0.123 \\
\hline Bt2 & $81-119$ & 300 & 100 & 400 & 150 & 400 & C & 1.48 & 44.15 & 14.86 & 0.24 & 0.115 & 0.123 \\
\hline Bt3 & $119-195$ & 400 & 100 & 500 & 100 & 400 & $\mathrm{SC}$ & 1.55 & 41.5 & 14.8 & 0.237 & 0.115 & 0.122 \\
\hline Mean & & 440 & 98 & 538 & 128 & 326 & & 1.40 & 47.01 & 13.95 & 0.229 & 0.111 & 0.117 \\
\hline $\mathrm{CV}(\%)$ & & 25.9 & 18.3 & 22 & 21.7 & 36.1 & & 9.5 & 10.7 & 13.4 & 5.8 & 4.9 & 6.6 \\
\hline$\overline{S E}$ & & 11.4 & 17.89 & 118.4 & 27.7 & 25.9 & & 0.13 & 5.04 & 1.88 & 0.01 & 0.01 & 0.01 \\
\hline
\end{tabular}

Table 6: Soil Physical Properties of Ovim, Abia State Profile

5.6. Soil Chemical Properties of Ikwuano, Umuahia Abia State Profile

Results in Table 7 showed the chemical properties of soils in Ikwuano, Abia State. The soils are strongly acidic with mean $\mathrm{pH}$ value of 4.56 in $\mathrm{KCl}$ and 5.3 in water. The mean values of organic carbon, organic matter, and total nitrogen were 9.6, 16.52 and 0.9 g.kg respectively. These values were low according to FAO (2006) rating. Exchangeable Ca, Mg, K and Na were low with mean value of total exchangeable bases of $1.56 \mathrm{cmol} / \mathrm{kg}$. Effective cation exchange capacity and base saturation were low. Available phosphorus was low with mean value of $20.68 \mathrm{mg} / \mathrm{kg}$. There was low variability in soil pH, exchangeable $\mathrm{Ca}$, total exchangeable bases, exchangeable $\mathrm{H}, \mathrm{Al}, \mathrm{TEA}, \mathrm{ECEC}$ and base saturation. Organic matter, total nitrogen, organic carbon and exchangeable $\mathrm{K}$ had high variation while exchangeable $\mathrm{Mg}$, Na and available $\mathrm{P}$ had medium variation.

\begin{tabular}{|c|c|c|c|c|c|c|c|c|c|c|c|c|c|c|c|c|c|c|}
\hline $\begin{array}{l}\text { : } \\
\text { : } \\
\text { : }\end{array}$ & 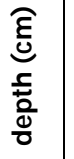 & $\begin{array}{l}\hat{\theta} \\
\text { 靣 }\end{array}$ & 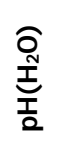 & 8 & $\Sigma$ & Z & $\delta$ & $\sum^{\infty}$ & $\forall$ & 2 & 用 & $I$ & ₹ & $\mathbb{1}$ & 8 & 㝗 & 莺 & $\frac{4}{4}$ \\
\hline & & & & $\mathrm{g} / \mathrm{kg}$ & $\mathrm{g} / \mathrm{kg}$ & $\mathbf{g} / \mathbf{k g}$ & 4 & & & & $\mathrm{Cmol} / \mathrm{kg}$ & & & & & $\rightarrow$ & $\%$ & $\mathrm{Mg} / \mathrm{kg}$ \\
\hline$\varangle$ & $\begin{array}{l}\overrightarrow{1} \\
\overrightarrow{1} \\
0\end{array}$ & $\stackrel{\infty}{+}$ & $\stackrel{0}{\dot{\omega}}$ & $\stackrel{\infty}{\sim}$ & $\vec{m}$ & $\stackrel{\varphi}{+}$ & $F$ & $\stackrel{+1}{0}$ & $\stackrel{8}{0}$ & 范 & ्ְ? & - & กิ & ๗ָ & 离 & ڤి & 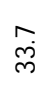 & ஓి \\
\hline$\frac{m}{4}$ & $\begin{array}{l}\infty \\
\text { p } \\
1 \\
\Rightarrow\end{array}$ & $\stackrel{\leftrightarrow}{+}$ & กี่ & $\underset{\sim}{ }$ & $\stackrel{\vec{H}}{\mathrm{H}}$ & $\stackrel{m}{\rightarrow}$ & -1 & m. & $\stackrel{\text { L̊ }}{\circ}$ & $\stackrel{\text { L̊ }}{\circ}$ & 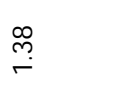 & $\stackrel{\text { T }}{7}$ & $\stackrel{m}{i}$ & $\stackrel{\varphi}{m}$ & 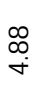 & 号 & $\stackrel{m}{\infty}$ & 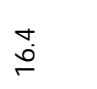 \\
\hline$\vec{艹}$ & 's 녕 & $\stackrel{\odot}{+}$ & ஜூ & $a$ & $\begin{array}{l}\stackrel{\circ}{\circ} \\
\stackrel{\rho}{\circ}\end{array}$ & $\ddot{\circ}$ & $\exists$ & $\stackrel{\text { L̊ }}{0}$ & $\stackrel{0}{0}$ & $\stackrel{0}{0}$ & $\underset{\sim}{\stackrel{N}{S}}$ & - & N & $m$ & $\underset{+}{\stackrel{\sim}{+}}$ & 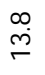 & $\begin{array}{l}+1 \\
\stackrel{m}{0}\end{array}$ & ֻั \\
\hline 苞 & 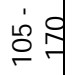 & $\underset{+}{\stackrel{\leftrightarrow}{+}}$ & में & กิ่ & $\infty$ & $\ddot{\circ}$ & 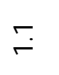 & म! & $\stackrel{8}{0}$ & $\stackrel{8}{0}$ & $\overrightarrow{\breve{r}}$ & - & N & $m$ & $\vec{\leftrightarrow}$ & $\underset{\vec{H}}{\stackrel{+}{*}}$ & 岗 & ה \\
\hline u & 웍뭥 & $\stackrel{+}{+}$ & n & $\stackrel{\infty}{\rightarrow}$ & $\vec{m}$ & $\stackrel{m}{0}$ & $r$ & $\stackrel{+1}{0}$ & $\stackrel{\%}{0}$ & $\stackrel{\%}{0}$ & $\stackrel{\mathscr{f}}{\sharp}$ & $\Xi$ & กิ & $\stackrel{m}{m}$ & 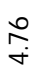 & $\underset{\dot{m}}{\dot{m}}$ & 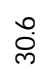 & $\stackrel{\infty}{\stackrel{\infty}{+}}$ \\
\hline $\begin{array}{l}\text { 粂 } \\
\sum_{2}\end{array}$ & & $\begin{array}{l}\stackrel{0}{h} \\
\stackrel{\leftrightarrow}{\not}\end{array}$ & $\stackrel{m}{i}$ & $\stackrel{\circ}{\circ}$ & 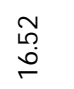 & ?. & $\underset{i}{\stackrel{\leftrightarrow}{i}}$ & 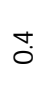 & $\begin{array}{l}0 \\
ٌ 0 \\
ٌ 0 \\
0\end{array}$ & $\begin{array}{l}\stackrel{D}{0}^{\infty} \\
\text { Oे }\end{array}$ & & 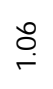 & $\stackrel{H}{\Delta}$ & $\ddot{\sim}$ & 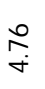 & $\begin{array}{l}\stackrel{\infty}{m} \\
\underset{+}{+}\end{array}$ & 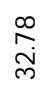 & $\begin{array}{l}\stackrel{0}{0} \\
\stackrel{\leftrightarrow}{\leftrightarrow} \\
\end{array}$ \\
\hline$\zeta \mathrm{d}^{2}$ & & $\hat{m}$ & $\stackrel{\sim}{\sim}$ & 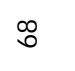 & $\stackrel{0}{\infty}$ & $\stackrel{+}{\mathrm{i}}$ & กี่ & $\stackrel{\curvearrowright}{\stackrel{\sim}{\sim}}$ & 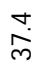 & $\stackrel{\sim}{\stackrel{\sim}{N}}$ & $\begin{array}{l}\infty \\
\infty \\
\infty\end{array}$ & $\stackrel{+}{+1}$ & $\dddot{m}$ & $\stackrel{\bullet}{\circ}$ & กิ & $\stackrel{0}{\circ}$ & 우 & $\begin{array}{l}\infty \\
\stackrel{\infty}{\infty} \\
\sim\end{array}$ \\
\hline 덩 & & $\stackrel{5}{\circ}$ & సิ & חొ & $\stackrel{\stackrel{n}{\rightarrow ~}}{\exists}$ & 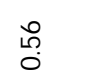 & $\stackrel{\circ}{0}$ & ô. & Oิ. & : & $\stackrel{H}{0}$ & : & $\stackrel{m}{0}$ & तิ & 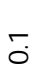 & 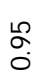 & ने & $\begin{array}{l}\text { 号 } \\
\text { เి }\end{array}$ \\
\hline
\end{tabular}

Table 7: Soil Chemical Properties Of Ikwuano, Umuahia Abia State Profile 


\subsection{Soil Chemical Properties of Obuzo, Abia State Profile}

Soil chemical properties of Obuzo, Abia State are presented in Table 8. The Ph level of the soils from A to Bt4 horizon is strongly acidic. Higher organic carbon and organic matter are found at the epipedon layer and this is in line with the results of other locations studied. However, the mean of organic carbon $(10.33 \mathrm{~g} / \mathrm{kg})$ and organic matter $(17.77 \mathrm{~g} / \mathrm{kg})$ are low using FAO (2006) rating standard. Both total nitrogen, exchangeable bases ( $\mathrm{Ca}, \mathrm{Mg}, \mathrm{K}, \mathrm{Na}$ ) are low and this could be attributed to low organic matter in the soil. Total exchangeable base was higher at the epipedon and this could be attributed the higher organic matter content at that layer. The mean effective cation exchange capacity $(6.30 \mathrm{cmol} / \mathrm{kg}) \mathrm{was}$ low while base saturation and available phosphorus were low (Esu, 1999). Variations also are observed in the properties with organic matter and organic carbon, total nitrogen, exchangeable sodium, and available phosphorus having high variability down the horizons.

\begin{tabular}{|c|c|c|c|c|c|c|c|c|c|c|c|c|c|c|c|c|c|c|}
\hline 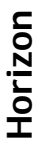 & 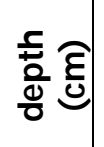 & 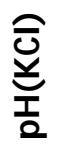 & 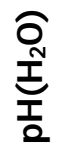 & 8 & $\sum$ & Z & 8 & $\sum$ & $\forall$ & Z & 鼻 & $\Phi$ & Z & 绿 & 置 & $\frac{8}{4}$ & 苟 & $\frac{a}{2}$ \\
\hline & & & & g/ kg & g/ kg & $\mathbf{g} / \mathbf{k g}$ & 4 & & & - & $\mathrm{Cmol} / \mathrm{kg}$ & & & & & $\rightarrow$ & $\%$ & $\mathrm{Mg} / \mathrm{kg}$ \\
\hline$\varangle$ & $\begin{array}{l}\stackrel{1}{7} \\
1 \\
0\end{array}$ & $\hat{m}$ & $\stackrel{\infty}{+\infty}$ & $\stackrel{\infty}{N}$ & 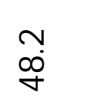 & $\stackrel{r}{-i}$ & $\stackrel{\varphi}{-}$ & $\stackrel{1}{0}$ & กָ & $\stackrel{0}{\circ}$ & $\begin{array}{l}\text { p) } \\
\text { in }\end{array}$ & نُّ & $N$ & 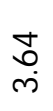 & 0 & $\infty$ & के & กै \\
\hline 量 & $\begin{array}{l}\stackrel{\infty}{N} \\
1 \\
\stackrel{1}{\sim}\end{array}$ & $\stackrel{\circ}{+}$ & 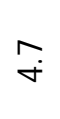 & $\stackrel{m}{\rightarrow}$ & $\stackrel{+}{\mathrm{N}}$ & $\stackrel{-}{-}$ & $\stackrel{-}{-}$ & $\dddot{m}$ & $\stackrel{0}{\stackrel{1}{0}}$ & $\stackrel{\text { Oे. }}{\circ}$ & 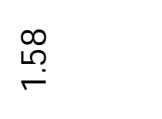 & $N$ & $\underset{N}{N}$ & 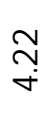 & $\begin{array}{l}\infty \\
\stackrel{\infty}{\circ}\end{array}$ & $\begin{array}{l}\text { N̦} \\
\infty\end{array}$ & ล & $\stackrel{N}{\Im}$ \\
\hline 呄 & $\begin{array}{l}\overleftrightarrow{\mid} \\
1 \\
\infty \\
\stackrel{1}{N}\end{array}$ & $\stackrel{N}{+}$ & $\stackrel{9}{+}$ & $\stackrel{ }{-1}$ & $\stackrel{\stackrel{N}{~}}{\sim}$ & $\begin{array}{l}0 \\
\text { H! }\end{array}$ & $\stackrel{\varphi}{-}$ & $\stackrel{100}{0}$ & กָ & $\overbrace{0}^{+1}$ & $\stackrel{H}{\text { N̦ }}$ & : & $m$ & $\begin{array}{l}\infty \\
\infty \\
+\end{array}$ & $\stackrel{N}{N}$ & 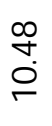 & లె & 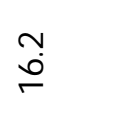 \\
\hline 志 & $\begin{array}{l}\infty \\
\infty \\
1 \\
1 \\
\qquad 1\end{array}$ & $\stackrel{N}{*}$ & ㅇ. & $\stackrel{\circ}{\circ}$ & $\stackrel{\sim}{\sim}$ & $\underset{H}{F}$ & $\stackrel{+}{-4}$ & $\stackrel{\pi}{0}$ & กุ & $\begin{array}{l}\mathscr{0} \\
0 \\
0\end{array}$ & $\begin{array}{l}\mathscr{\ell} \\
\text { i }\end{array}$ & $\underset{\text { Oे }}{\text { ì }}$ & مِ & ஸे & $\stackrel{\varphi}{\stackrel{\varphi}{\circ}}$ & $\infty$ & ம & $\stackrel{0}{0}$ \\
\hline 䓟 & $\begin{array}{l}\stackrel{0}{\circ} \\
\stackrel{1}{1} \\
1 \\
\infty \\
\infty\end{array}$ & $\overrightarrow{+}$ & $\stackrel{9}{+}$ & $\stackrel{\circ}{\dot{m}}$ & ம் & $\begin{array}{l}\stackrel{\infty}{N} \\
0\end{array}$ & $\stackrel{\text { N }}{ }$ & $\hat{0}$ & $\stackrel{m}{0}$ & $\begin{array}{l}\infty \\
0 \\
0\end{array}$ & $\begin{array}{l}\stackrel{\infty}{N} \\
\text { nd }\end{array}$ & $\vec{i}$ & $\begin{array}{l}\vec{\infty} \\
\stackrel{N}{N}\end{array}$ & $\underset{+}{\text { ठ઼ }}$ & No & $\underset{\sim}{\sim}$ & 암 & $\stackrel{+}{\circ}$ \\
\hline 苟 & $\begin{array}{l}\stackrel{8}{8} \\
\stackrel{7}{1} \\
1 \\
\stackrel{\circ}{\circ}\end{array}$ & $\stackrel{\dot{\leftrightarrow}}{+}$ & $\stackrel{6}{+i}$ & $\stackrel{\circ}{\rightarrow}$ & i & $\underset{\sigma}{ت}$ & $\stackrel{-}{-}$ & $\dddot{m}$ & $\begin{array}{l}\infty \\
\stackrel{1}{0}\end{array}$ & $\underset{0}{0}$ & $\stackrel{0}{\sim}$ & $\stackrel{-}{-}$ & $\stackrel{m}{i}$ & $\stackrel{+}{\oplus}$ & $\stackrel{\circ}{\circ}$ & $\underset{ }{0}$ & లె & $\stackrel{0}{0}$ \\
\hline $\begin{array}{l}\mathbb{\pi} \\
\sum_{\Sigma}^{\infty}\end{array}$ & & 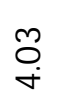 & & $\begin{array}{l}m \\
\stackrel{m}{0} \\
\stackrel{\sigma}{0}\end{array}$ & $\stackrel{尺}{\stackrel{ }{\sim}}$ & \begin{tabular}{l}
$\infty$ \\
\hdashline \\
0
\end{tabular} & $\stackrel{\circ}{\rightarrow}$ & $\stackrel{\text { f? }}{0}$ & స̦ & $\stackrel{\mathscr{L}}{\circ}$ & $\stackrel{\text { N. }}{\text {. }}$ & $\stackrel{?}{\rightarrow}$ & $\overrightarrow{\mathrm{j}}$ & $\stackrel{\circ}{\stackrel{ }{+}}$ & $\begin{array}{l}\stackrel{\Re}{0} \\
\text { }\end{array}$ & $\begin{array}{l}\text { Lొ } \\
\text { ஸึ. }\end{array}$ & $\begin{array}{l}m \\
m \\
\ddot{m}\end{array}$ & $\stackrel{\hat{m}}{+}$ \\
\hline$\frac{a}{8}$ & & $\stackrel{\varphi}{+}$ & $\vec{m}$ & ठే & ֻै & ஸి & 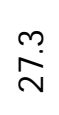 & $\hat{m}$ & $\stackrel{\leftrightarrow !}{N}$ & $\frac{9}{1}$ & $\begin{array}{l}\stackrel{\llcorner}{0} \\
\stackrel{\infty}{N}\end{array}$ & $\vec{N}$ & カ艹 & $\begin{array}{l}\stackrel{\leftrightarrow}{\rho} \\
\stackrel{\infty}{N}\end{array}$ & $\stackrel{\wedge}{\infty}$ & $\stackrel{N}{N}$ & $\underset{\sim}{\stackrel{m}{\sim}}$ & H' \\
\hline 嵌 & & $\stackrel{9}{\rightarrow}$ & $\stackrel{+}{\stackrel{H}{0}}$ & $\hat{\sigma}$ & $\begin{array}{l}m \\
\stackrel{0}{6} \\
0\end{array}$ & $\stackrel{0}{0}$ & $\underset{0}{\vec{H}}$ & $\stackrel{\text { L }}{\stackrel{0}{0}}$ & $\begin{array}{l}\text { O’ } \\
\end{array}$ & Oै. & 包 & $\begin{array}{l}\text { ले } \\
0\end{array}$ & 苞 & ्ְठ & $\stackrel{\substack{\rightarrow \\
\rightarrow}}{\rightarrow}$ & $\overrightarrow{\widehat{N}}$ & $\begin{array}{l}\text { Oे } \\
+\end{array}$ & $\begin{array}{l}\stackrel{\leftrightarrow}{N} \\
\text { on }\end{array}$ \\
\hline
\end{tabular}

Table 8: Soil Chemical Properties of Obuzo, Abia State Profile

\subsection{Soil Chemical Properties of Ovim, Abia State Profile}

The chemical properties of Ovim soil in Abia State are presented in Table 9. The soils are moderately acidic with low variability. Organic carbon and organic matter are low using FAO (2006) fertility rating. High variations were observed in the organic carbon, organic matter and total nitrogen content within the horizons. This could be due to litter fall influence on soil organic matter on the epipedon which decreases down the pit. The mean values of organic carbon, organic matter and total nitrogen were 10.60, 18.24 and $0.82 \mathrm{~g} / \mathrm{kg}$ respectively. According to FAO, (2006), exchangeable 
$\mathrm{Ca}, \mathrm{Mg}, \mathrm{K}$ and Na were low with moderate variability. Effective cation exchange capacity was low with mean value of $3.53 \mathrm{cmol} / \mathrm{kg}$. However, base saturation was moderate and available phosphorus was low with medium variability.

\begin{tabular}{|c|c|c|c|c|c|c|c|c|c|c|c|c|c|c|c|c|c|c|}
\hline 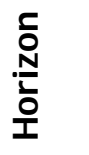 & $\frac{\frac{\pi}{2}}{\frac{2}{d}}$ & $\begin{array}{l}\hat{8} \\
\text { 20 }\end{array}$ & 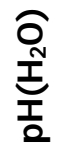 & 8 & 要 & Z & 8 & $\sum$ & 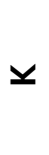 & Z & 盖 & $\boldsymbol{I}$ & Z & 畐 & 导 & 8 & $\overrightarrow{8}$ & $\frac{4}{4}$ \\
\hline & & & & $\begin{array}{c}g / k \\
g\end{array}$ & $\begin{array}{c}g / k \\
g\end{array}$ & $\begin{array}{c}g / k \\
g\end{array}$ & 4 & & & - & $\begin{array}{c}\mathrm{Cmol} / \mathrm{k} \\
\mathrm{g}\end{array}$ & & & & & $\rightarrow$ & $\%$ & $\begin{array}{c}\mathrm{Mg} / \mathrm{k} \\
\mathrm{g}\end{array}$ \\
\hline$\varangle$ & $\begin{array}{l}\text { ㄱ } \\
1 \\
0\end{array}$ & $\stackrel{+H}{+}$ & $\overrightarrow{10}$ & $\stackrel{M}{N}$ & $\begin{array}{l}\text { @े } \\
\text { ले }\end{array}$ & $\stackrel{\varphi}{-}$ & $\stackrel{\infty}{0}$ & $\stackrel{0}{0}$ & $\begin{array}{l}\text { లి } \\
\text { లె }\end{array}$ & $\overrightarrow{7}$ & $\stackrel{\widehat{\infty}}{-\infty}$ & $\stackrel{M}{\rightarrow}$ & $\stackrel{\sim}{\sim}$ & $\stackrel{m}{\text { mे }}$ & $\stackrel{\sim}{+}$ & $\stackrel{\stackrel{H}{N}}{N}$ & $\stackrel{\llcorner}{\forall}$ & 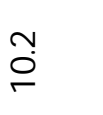 \\
\hline 旪 & $\begin{array}{l}\text { F } \\
1 \\
\stackrel{1}{-}\end{array}$ & $\stackrel{-}{+}$ & L & $\stackrel{m}{\rightarrow-1}$ & $\stackrel{\stackrel{H}{*}}{\stackrel{H}{N}}$ & $\stackrel{M}{-}$ & $\stackrel{\text { L̊ }}{0}$ & $\dddot{m}$ & $\stackrel{\infty}{\stackrel{1}{0}}$ & 8 & 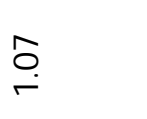 & $r$ & $\stackrel{m}{r}$ & $\stackrel{m}{\stackrel{m}{i v}}$ & $\stackrel{\infty}{\infty}$ & छै & 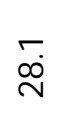 & تే \\
\hline 苟 & \begin{tabular}{l}
$\vec{\infty}$ \\
1 \\
\multirow{y}{*}{}
\end{tabular} & $\stackrel{\llcorner}{\not+}$ & $\vec{\omega}$ & $a$ & 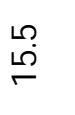 & $\stackrel{0}{0}$ & $\hat{0}$ & ְ̣̊ & $\stackrel{+}{0}$ & $\ddot{0}$ & $\hat{-}$ & $r$ & $\stackrel{N}{\sim}$ & กั & $\ddot{\leftrightarrow}$ & $\widehat{0}$ & 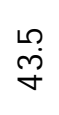 & $\sigma$ \\
\hline 苚 & $\begin{array}{l}\underset{-1}{-1} \\
\underset{1}{1} \\
-\infty\end{array}$ & F & $\begin{array}{l}6 \\
\stackrel{0}{\circ}\end{array}$ & م & $\begin{array}{l}0 \\
\infty\end{array}$ & ?ִ & $\stackrel{0}{0}$ & $\stackrel{0}{0}$ & $\stackrel{m}{0}$ & $\ddot{0}$ & $\stackrel{0}{-}$ & $\stackrel{\text { न }}{\rightarrow}$ & -1 & $\overrightarrow{\mathrm{i}}$ & $\begin{array}{l}\hat{\curvearrowright} \\
\text { ले }\end{array}$ & คి & $\stackrel{\stackrel{H}{+}}{\stackrel{f}{+}}$ & $\stackrel{\infty}{\infty}_{\infty}$ \\
\hline$\stackrel{M}{\dot{M}}$ & $\begin{array}{l}\stackrel{\Omega}{2} \\
\stackrel{1}{1} \\
\stackrel{2}{=}\end{array}$ & $\stackrel{0}{+}$ & مึ & $m$ & $\overrightarrow{10}$ & -1 & $\stackrel{m}{0}$ & $\dddot{m}$ & กิ & $\stackrel{\infty}{\infty}$ & $\ddot{0}$ & $\stackrel{\llcorner}{0}$ & $\stackrel{0}{0}$ & $\overrightarrow{-}$ & $N$ & $\begin{array}{l}\mathscr{8} \\
\text { m. }\end{array}$ & $\stackrel{10}{+}$ & $\stackrel{+}{+}$ \\
\hline 蛋 & & $\stackrel{\substack{1 \\
+}}{+}$ & $\begin{array}{l}\stackrel{0}{N} \\
\text { ம் }\end{array}$ & $\underset{0}{\stackrel{0}{0}}$ & $\underset{\substack{+\sim}}{\sim}$ & $\stackrel{1}{\infty}$ & $\begin{array}{l}\infty \\
\stackrel{1}{1} \\
0\end{array}$ & $\stackrel{0}{\ddagger}$ & ণิ & $\stackrel{0}{-1}$ & $\stackrel{\mathscr{P}}{\rightarrow}$ & \%ุ & $\stackrel{\text { L }}{\sim}$ & $\overrightarrow{\vec{i}}$ & חึ & $\begin{array}{l}\infty \\
0 \\
0\end{array}$ & $\begin{array}{l}\text { R } \\
\text { o }\end{array}$ & $\stackrel{N}{\stackrel{N}{\curvearrowright}}$ \\
\hline$\overline{0}$ & & ก้ & $\overrightarrow{1}$ & $\stackrel{\infty}{\mathbb{H}^{+}}$ & $\stackrel{\circ}{+}$ & $\stackrel{\circ}{+}$ & $\stackrel{m}{m}$ & m & $\stackrel{\varphi}{\dot{m}}$ & $\stackrel{9}{-}$ & ஓ্ণ & 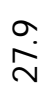 & $\begin{array}{l}\dot{\varphi} \\
\stackrel{\leftrightarrow}{m}\end{array}$ & 灾 & ঙ્H & $\vec{\lambda}$ & 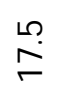 & ஷ্ণ \\
\hline 䍖 & & $\stackrel{\text { }}{\text { N̦ }}$ & సิ & ๙઼ & $\stackrel{\widehat{\infty}}{\stackrel{N}{o}}$ & 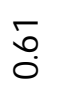 & $\stackrel{9}{-1}$ & $\stackrel{10}{\circ}$ & $\stackrel{8}{\circ}$ & -1. & $\stackrel{\text { f }}{0}$ & กิ & $\overrightarrow{7}$ & $\stackrel{0}{0}$ & $\begin{array}{c}\hat{\infty} \\
0 \\
0\end{array}$ & $\stackrel{\substack{n \\
\sim}}{\sim}$ & $\stackrel{ }{\overparen{r}}$ & $\overrightarrow{\mathrm{m}}$ \\
\hline
\end{tabular}

Table 9: Soil chemical properties of Ovim, Abia State profile

\subsection{Comparison of Soil Physical and Chemical Properties within Two Sampling Depths of Abia State}

Results in Table 10 and 11 showed the comparison in the physicochemical properties of soils in Abia State between the two sampling depths. To compare the similarity to the result in some other states like Ebony and Imo, there was significant effect $(p=0.05$ ) on soil bulk density and total porosity. There were no significant effects on soil chemical properties except exchangeable $\mathrm{H}$, potassium and base saturation. 


\begin{tabular}{|c|c|c|c|c|c|c|c|c|c|c|c|c|}
\hline 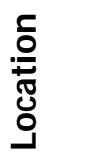 & 혈 웡 & 8 & I & 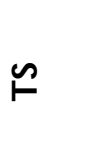 & is & $\sigma$ & 要 & $\hat{H}$ & $\Xi$ & U & $\sum_{2}^{0}$ & 这 \\
\hline & & $\mathrm{g} / \mathrm{kg}$ & $\mathrm{g} / \mathrm{kg}$ & $\mathrm{g} / \mathrm{kg}$ & $\mathrm{g} / \mathrm{kg}$ & $\mathrm{g} / \mathrm{kg}$ & & $\mathrm{g} / \mathrm{cm}^{3}$ & $\%$ & $\%$ & $\mathrm{~g} / \mathrm{kg}$ & $\mathrm{g} / \mathrm{kg}$ \\
\hline $\begin{array}{l}\frac{\pi}{\pi} \\
\frac{\pi}{4}\end{array}$ & l & $\stackrel{\dot{\theta}}{\vec{\gamma}} \stackrel{\infty}{\sim}$ & $\stackrel{\mathscr{N}}{\stackrel{\circ}{N}}$ & $\stackrel{\ddot{\sim}}{\nabla} \ddot{m}$ & 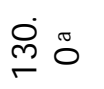 & $\stackrel{\dot{D}}{\sim} \underset{\sim}{\sim}$ & $\stackrel{\stackrel{H}{+}}{-} \sigma$ & $\tilde{n}_{\tilde{n}}^{\infty} \sigma$ & مْ & $\vec{\pi}_{0}$ & $\stackrel{\circ}{\stackrel{-}{0}}$ & $\vec{ت}$ \\
\hline \multirow[t]{2}{*}{ 丞离 } & 온 아 & $\begin{array}{l}\hat{\oplus} \\
\stackrel{\leftrightarrow}{\gamma}\end{array}$ & $\begin{array}{l}\stackrel{0}{0} \\
\stackrel{\vec{m}}{m}\end{array}$ & $\begin{array}{l}\hat{0} \\
\hat{D}\end{array}$ & 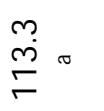 & $\begin{array}{l}\stackrel{0}{\circ} \\
\stackrel{\circ}{\circ}\end{array}$ & $\stackrel{\text { ח̊ }}{\text { mె }}$ & مـ & $\underset{\circlearrowright}{0}$ & 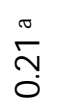 & $\begin{array}{l}\stackrel{\pi}{8} \\
\stackrel{0}{0}\end{array}$ & $\stackrel{\sim}{\sim}$ \\
\hline & कृष्ठ & $\begin{array}{l}+ \\
\infty \\
\infty \\
\infty \\
m\end{array}$ & $\stackrel{\hat{\rho}}{\stackrel{\hat{\rho}}{-}}$ & 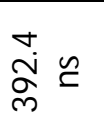 & 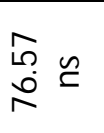 & 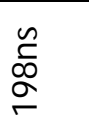 & $\stackrel{\infty}{\infty}$ & $\begin{array}{l}\infty \\
\infty \\
i \mathfrak{j}\end{array}$ & $\begin{array}{l}\mathscr{0} \\
\stackrel{0}{0} \\
\stackrel{0}{0}\end{array}$ & 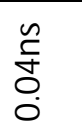 & 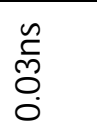 & 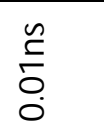 \\
\hline
\end{tabular}

Table 10: Comparison of Soil Physical Properties within Two Sampling Depths of Abia State

Means having the same letters are not significant at 0.05 probability level, ns = not significant

\begin{tabular}{|c|c|c|c|c|c|c|c|c|c|c|c|c|c|c|c|c|c|c|}
\hline 을 & 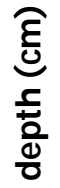 & 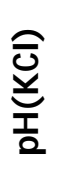 & 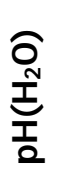 & 8 & $\sum$ & Z & $\mathscr{8}$ & $\sum^{\infty}$ & $\forall$ & Z & 䚁 & $\mathbf{I}$ & ¿ & 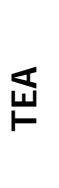 & 亩 & 导 & $\overrightarrow{8}$ & $\frac{4}{4}$ \\
\hline & & & & $\mathrm{g} / \mathrm{kg}$ & g/ kg & g/ kg & 4 & & & - & $\mathrm{Cmol} / \mathrm{kg}$ & & & & & $\rightarrow$ & $\%$ & $\mathrm{Mg} / \mathbf{k g}$ \\
\hline 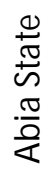 & $\begin{array}{l}\stackrel{\curvearrowright}{N} \\
1 \\
0\end{array}$ & $\begin{array}{l}\text { סू } \\
\text { ले }\end{array}$ & $\begin{array}{c}\tilde{N} \\
\stackrel{+}{+}\end{array}$ & 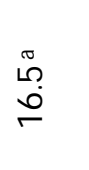 & $\begin{array}{l}\stackrel{\infty}{H} \\
\stackrel{+}{\infty} \\
\stackrel{\infty}{N}\end{array}$ & 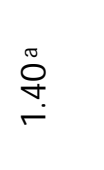 & $\stackrel{\pi}{\hat{\sigma}}$ & $\begin{array}{l}\stackrel{0}{0} \\
\text { ஸे } \\
0\end{array}$ & $\begin{array}{l}0 \\
0 \\
0\end{array}$ & $\stackrel{\pi}{\stackrel{\pi}{0}}$ & $\stackrel{\text { m̦ }}{\text { m̦ }}$ & 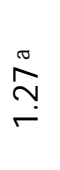 & $\stackrel{\pi}{\stackrel{0}{0}}$ & $\begin{array}{l}\stackrel{\infty}{M} \\
\stackrel{m}{m}\end{array}$ & 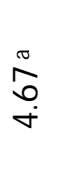 & $\begin{array}{l}\stackrel{0}{0} \\
\stackrel{0}{0} \\
0\end{array}$ & $\begin{array}{l}\stackrel{0}{0} \\
\hat{N}\end{array}$ & $\begin{array}{l}\tilde{N} \\
\stackrel{\infty}{\infty} \\
\infty \\
\rightarrow\end{array}$ \\
\hline 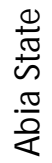 & $\begin{array}{l}\text { ㅇ } \\
\text { l } \\
\text { ㅅ }\end{array}$ & $\begin{array}{l}\stackrel{\sigma}{R} \\
\text { m }\end{array}$ & $\begin{array}{l}\stackrel{\beta}{\hat{\beta}} \\
\text { mi }\end{array}$ & $\begin{array}{l}\text { ஸ̃ } \\
\text { ஸे }\end{array}$ & 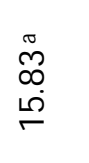 & $\stackrel{\tilde{n}}{\stackrel{\sigma}{0}}$ & $\stackrel{0}{0}_{0}^{0}$ & $\stackrel{\sigma}{\circ} \stackrel{0}{0}$ & 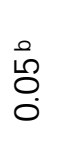 & $\stackrel{\tilde{\sigma}}{\stackrel{\sigma}{0}}$ & $\stackrel{\pi}{\stackrel{0}{\hat{0}}}$ & 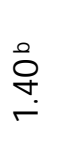 & 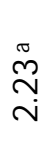 & $\begin{array}{l}\tilde{\pi} \\
\ddot{\leftrightarrow} \\
\dot{m}\end{array}$ & 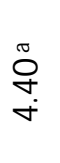 & $\begin{array}{l}\underset{f}{\tilde{H}} \\
\stackrel{0}{0}\end{array}$ & $\begin{array}{l}\stackrel{2}{\hat{\theta}} \\
\stackrel{0}{\sigma}\end{array}$ & $\begin{array}{l}\stackrel{\pi}{-} \\
\stackrel{-}{-}\end{array}$ \\
\hline ڤ్ & & 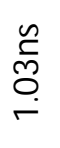 & $\stackrel{\substack{-1 \\
\hdashline}}{0}$ & 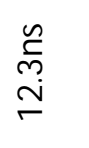 & 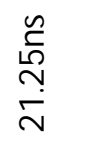 & $\begin{array}{l}\text { है } \\
\text { है } \\
\dot{0}\end{array}$ & $\begin{array}{l}\text { ?్ర } \\
\text { ț } \\
0\end{array}$ & 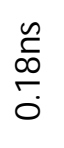 & $\stackrel{1}{0}$ & 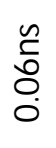 & $\begin{array}{l}0 \\
00 \\
0 \\
0 \\
0\end{array}$ & $\begin{array}{l}\infty \\
\stackrel{-}{0}\end{array}$ & $\frac{\text { בै }}{\stackrel{2}{0}}$ & 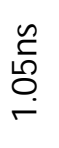 & 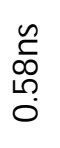 & $\begin{array}{l}\underset{c}{c} \\
\stackrel{1}{0} \\
\stackrel{-}{-}\end{array}$ & $\stackrel{m}{\sim}$ & 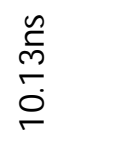 \\
\hline
\end{tabular}

Table 11: Comparison of Soil Chemical Properties within Two Sampling Depths of Abia State

Means having the same letters are not significant at 0.05 probability level, ns = not significant

5.10. Relationship between Edaphic Factors and Crop Yield in Abia State

Results of the relationship between edaphic factors and crop yield as shown in Table 12 revealed that there is a relationship between soil (edaphic) factors and crop yield. Cassava yield significantly correlated negatively with edaphic factors like anion exchange capacity, available P, CS, and exchangeable Ca, effective cation exchange capacity, total exchangeable bases and TS but significantly correlated negatively with bulk density and FC.

Similarly, maize yield significantly correlated negatively with bulk density and base saturation but significantly correlated positively with anion exchange capacity, available P, base saturation, exchangeable $\mathrm{Ca}$, FS, exchangeable Mg, organic carbon, organic matter, total exchangeable bases, total nitrogen and soil $\mathrm{pH}$. These results also confirmed the observation made in Imo State that edaphic factors influence cassava and maize yield. 


\begin{tabular}{|c|c|c|}
\hline Parameter & Cassava yield & Maize yield \\
\hline Cassava yield & 1 & \\
\hline Maize yield & $-0.8913^{* *}$ & 1 \\
\hline Average Rainfall & $0.5939 *$ & $-0.8567 * *$ \\
\hline Average Temp & $-0.7141 * *$ & $0.6872^{*}$ \\
\hline Moisture content & -0.1695 & 0.2656 \\
\hline Anion Exchange Capacity & $-0.6662 *$ & 0.5693* \\
\hline Available water capacity (AWC) & 0.0613 & -0.3529 \\
\hline Exchangeable Al & -0.447 & 0.1883 \\
\hline Available Phosphorus & $-0.6393^{*}$ & $0.6846^{*}$ \\
\hline Bulk Density & $0.6505^{*}$ & $-0.8371^{* *}$ \\
\hline Base saturation & -0.3800 & $0.6500^{*}$ \\
\hline $\mathrm{CS}$ & $-0.6742^{*}$ & 0.3475 \\
\hline Exchangeable Ca & $-0.5883^{*}$ & $0.6826^{*}$ \\
\hline $\mathrm{Cl}$ & 0.0714 & -0.0275 \\
\hline Effective Cation Exchange Capacity & $-0.6382^{*}$ & $0.5719 *$ \\
\hline $\mathrm{FC}$ & $0.5020^{*}$ & -0.4197 \\
\hline FS & -0.2398 & $0.1550^{*}$ \\
\hline Exchangeable $\mathrm{H}$ & 0.3333 & -0.4758 \\
\hline Exchangeable K & -0.0567 & 0.3422 \\
\hline Exchangeable Mg & -0.4242 & $0.7407 * *$ \\
\hline Exchangeable Na & -0.0556 & 0.3451 \\
\hline Organic Carbon & -0.4406 & $0.7115^{* *}$ \\
\hline Organic Matter & -0.4404 & $0.7113^{* *}$ \\
\hline PWP & 0.2473 & 0.0524 \\
\hline $\mathrm{Si}$ & 0.1962 & -0.1021 \\
\hline Total Exchangeable Acidity & -0.3269 & 0.0505 \\
\hline Total Exchangeable bases & $-0.5595^{*}$ & $0.7366^{* *}$ \\
\hline Total N & -0.2746 & $0.6632 *$ \\
\hline Total Porosity & $-0.6500^{*}$ & $0.8367 * *$ \\
\hline TS & $-0.7795^{* *}$ & 0.4203 \\
\hline $\mathrm{pH}\left(\mathrm{H}_{2} \mathrm{O}\right)$ & -0.2537 & $0.5314^{*}$ \\
\hline
\end{tabular}

Table 12: Relationship between Edaphic Factors and Yield of Maize and Cassava in Abia State

*And** $=$ Sig at 0.05 and 0.01 Probability Levels Respectively

Source: Author's Field Work (2019)

\section{Conclusion}

Abia State is agrarian state by nature and its soil is needed to be improved in quality so as to boost agricultural productivity. The study show that soils of Abia state had a good correlation with the arable crops. This means that soils properties affects crop production in various ways, but other factors such as technology, environmental hazards, and farmers' adaptive skill to climatic variations and change can also account for variations recorded respectively in maize and cassava yields in the state. In the area, total sand (Ts) at top soil level is $713 \mathrm{~kg}$ and $737 \mathrm{~kg}$ at sub-soil level against silt (Si) with $130 \mathrm{~kg}$ at top soil level and $113 \mathrm{~kg}$ at sub soil level. The study however, indicated that the state is very rich in total sand, and the soils in the state are more porous and had more moisture content and field capacity. Previous research; Akamigbo (1984) reported that soils of Southern Nigeria are low in salt as a result of the high degree and extent of weathering and leaching have undergone. Similar results were also reported by Onweremadu and Anikwe (2007) while working on the soils of Isienyi Ibeku in Abia State. Onweremadu and Anikwe (2007) reported very low to moderate silt/ clay ratios ( 0.04 to 0.029$)$ that generally decreased with depth while working on soils at Isienyi Ibeku, which is within Ikwuano soil zone in Abia State. This study however reports high to very high silt/ clay ratios (1:9.8) in Ikwuano in general, and the ratios increased with depth. This observation could be attributed to different parent materials in the areas as well 
as land-use system and climatic conditions. Also for chemical properties, the state is dominated by exchangeable $\mathrm{Ca}, \mathrm{Mg}, \mathrm{K}$, $\mathrm{Na}$ as well as total exchangeable acidity. It also had higher values of ECEC, ACEC, base saturation and available phosphorus. Furthermore, it was observed that Abia state soil contains more plant nutrient elements that will support crop production. However, the chemical properties of soils in the state are still low according to FAO (2006); Esu (1991) soil fertility rating. Hence, there is therefore need to boost more the productivity of soils in the State for maximum crop production.

\section{Recommendations}

- Proper soil management strategies will be necessary for the farmers in the state to adopt in order to boost soils qualities for optimum food production.

- Abia state government needs to give incentives like organic fertilizers to the farmers which will support growth and development of food crops for yield maximization.

- Climate is another physical factor affecting soils quality; therefore, farmers need education and awareness on the climate risks, which reduces soil quality and in turn affects their crops.

\section{References}

i. Agbola, T and Ojeleye, D (2007): Climate change and food crop production in Ibadan, Nigeria. Afr. Crop Sci. Conf. Proc. 8: 1423-1433.

ii. Akamigbo, F.O.R. (1984): The accuracy of field texture in a humid tropical environment. Soil Survey and Land Evaluation 493.63-70

iii. Ani, A.O, (2002) Factors Inhibiting Agricultural Projection Among Rural Women Farmers in Southern Ebonyi State, Nigeria Ph.D. Thesis. University of Maiduguri, Nigeria.

iv. Apata, T.G, Samuel, K.D and Adeola, A.O (2009). Analysis of Climate Change Perception and Adaptation among Arable Food Crop Farmers in South Western Nigeria. Contributed Paper prepared for presentation at the International Association of Agricultural Economists' 2009 Conference, Beijing, China, 16-22 August 2009.

v. Bremner, J.M (1996) Nitrogen total. In: Sparus, D. L (ed) Methods of soil analysis part 3, Chemical methods, vol 5. Soil Sci. Soc. Am. Book series. Pp. 1085-1122.

vi. Esu, I.E (1991). Detailed soil survey of NIHORT farm at Bunkure, Kano State, Nigeria. Institute for Agricultural Research, Ahmadu Bello University, Zaria.

vii. FAO (Food and Agricultural Organization) (2006) Guidelines for soil description (4th ed.) Rome: Food and Agriculture Organization of the United Nations. 97pp.

viii. Foth, H. D (1984). Fundamentals of soil science. $7^{\text {th }}$ edition. John Wiley and Sons New York. 435Pp.

ix. Gee, G.W. and Or, D. (2002). Particle size analysis. In: Dane, J.H. and Topp, D.C (eds). Methods of soil analysis, Part 4. Physical methods, Soil sci. Soc. Am. Book series No.5 ASA and SSSA, Madison, W.I. Pp. 255-293.

x. Grossman, R.P. and Reinschi, T.G. (2002). Bulk density and linear extensibility. In: Dane, J.H. and Topp, D.C (eds). Methods of soil analysis, Part 4. Physical methods, Soil sci. Soc. Am. Book series No.5 ASA and SSSA, Madison, W.I. Pp. 201-228

xi. Hendershot, W.H., Laland, H. and Duquette, M (1993). Soil reaction and exchangeable acidity. In: Carter, M.R (ed). Soil sampling and methods of soil analysis. Canadian Soc. Soil Sci, Lewis Publishers, London. Pp. 141-145.

xii. International Panel on Climate Change (2007a): In Contribution of Working Groups I, II and III to the Fourth Assessment Report of the Intergovernmental Panel on Climate Change, Writing Team C, Pa-chauri RK, Reisinger A (eds). IPCC: Geneva; 104.

xiii. Lia, R (1979). Physical characteristic of soil in the Tropics; Determination and management in Lar R and Greenland, D. eds Willey and Sons. Uk

xiv. National Population Commission (NPC) (2006) Provisional Population Census Report. Abuja, National Bureau of Statistics.

xv. Njoku, J. D. (2006). Analysis of the Effect of Global Warming on Forests of Southeastern Nigeria using remotely Sensed Data. Unpublished, Ph.D Dissertation, Department of Geography and Environmental Management, Imo State University Owerri

xvi. Nnaji, G. U, Asadu C. and Mbagwu, J. S (2002). Evaluation of Physio-properties of soil under selected agricultural land utilization types. Journal of Tropical Agriculture, Food, environment and Extension Vol. 3 No 1:27-33pp

xvii. Olson, R.S., and Sommers, L.E (1982). Phosphorous. In Page, A.L., Miller, R.H. and Keeney, D.R. (eds). Methods of soil analysis. Am. Soc. Agron., Madison, W.I. Pp. 403-430.

xviii. Onweremadu, E.U and Anikwe, M.A.N. (2007) Soil wettability characteristics of a forested catena in relation to organic matter fractions. International journal of soil Science 2(3):211-217.

xix. Soil Survey Staff (2003) Keys to soil taxonomy (9th ed.) Washington, DC: Natural Resources Conservation Service, United States Department of Agriculture (USDA). 332pp.

xx. Soil Survey Staff (2010): Keys to Soil Taxonomy. 11 th Edition. USDA - NRCS. Washington, DC. 338pp

xxi. Wilding, L. P., Bouma, J. \& Boss, D. W. (1994) Impact of spatial variability on interpretative modeling, In: Bryant R. B. and R. W. Arnold: Quantitative Modeling of Soil Forming Process, SSSA Special Publication, vol. 39, pp. 61-75 


\section{Appendix}

Acronyms Used in This Study

Physical Soil Properties:

$\mathrm{CS}=$ Coarse Sand, $\mathrm{FS}=$ Fine Sand, $\mathrm{TS}=$ Total Sand, $\mathrm{Si}=$ Silt, $\mathrm{Cl}=$ Clay Sand, $\mathrm{TC}=$ Textural Class, $\mathrm{SL}=$ Sandy Loam, $\mathrm{SCL}=$ Sandy Clay Loam, SC = Sandy Clay, BD = Bulk Density, TP = Total Porosity, OM = Gravimetric Moisture Content, FC = Field Capacity, PWP = Permanent Wilting Point, AWC = Available Water Capacity.

Chemical Soil Properties:

$\mathrm{pH}(\mathrm{KCL})=\mathrm{pH}$ Potassium Chloride, $\mathrm{pH}(\mathrm{H} 20)=\mathrm{pH}$ water, $\mathrm{OC}=$ Organic Carbon, $\mathrm{OM}=$ Organic Matter, $\mathrm{TN}=$ Total Nitrogen, $\mathrm{Ca}=$ Calcium, $\mathrm{Mg}=$ Magnesium, $\mathrm{K}=$ Potassium, $\mathrm{Na}=$ Sodium, $\mathrm{TEB}=$ Total Exchangeable Base, $\mathrm{H}=$ Hydrogen ion, $\mathrm{Al}=$ Aluminium, TEA = Total Exchangeable Acidity, ECEC= Effective Cation Exchange Capacity, ACEA = Apparent Cation Exchangeable Capacity, Bsat = Base Saturation and Av. P = Available Phosphorous, $+=$ Exchangeable, SOC = Soil Organic Carbon, $\mathrm{SO}=$ Soil Organism, $\mathrm{CEC}=$ Cation Exchange capacity, EC = Electrical Conductivity, ESP $=$ Exchangeable Sodium Percentage, EPP = Exchangeable Potassium Percentage, $\mathrm{pH}=$ (Soil reaction) hydrogen ion concentration. 